\title{
Travelling convectons in binary fluid convection
}

\author{
Isabel Mercader ${ }^{1} \uparrow$, Oriol Batiste ${ }^{1}$, Arantxa Alonso ${ }^{1}$ and Edgar Knobloch ${ }^{2}$ \\ ${ }^{1}$ Departament de Física Aplicada, Universitat Politècnica de Catalunya, 08034 Barcelona, Spain \\ ${ }^{2}$ Department of Physics, University of California, Berkeley, CA 94720, USA
}

(Received 16 May 2012; revised 28 January 2013; accepted 4 February 2013; first published online 28 March 2013)

Binary fluid mixtures with a negative separation ratio heated from below exhibit steady spatially localized states called convectons for supercritical Rayleigh numbers. With no-slip, fixed-temperature, no-mass-flux boundary conditions at the top and bottom stationary odd- and even-parity convectons fall on a pair of intertwined branches connected by branches of travelling asymmetric states. In appropriate parameter regimes the stationary convectons may be stable. When the boundary condition on the top is changed to Newton's law of cooling the odd-parity convectons start to drift and the branch of odd-parity convectons breaks up and reconnects with the branches of asymmetric states. We explore the dependence of these changes and of the resulting drift speed on the associated Biot number using numerical continuation, and compare and contrast the results with a related study of the Swift-Hohenberg equation by Houghton \& Knobloch (Phys. Rev. E, vol. 84, 2011, art. 016204). We use the results to identify stable drifting convectons and employ direct numerical simulations to study collisions between them. The collisions are highly inelastic, and result in convectons whose length exceeds the sum of the lengths of the colliding convectons.

Key words: buoyancy-driven instability, double diffusive convection, pattern formation

\section{Introduction}

Spatially localized states occur in a variety of different physical systems including reaction-diffusion systems (Lee et al. 1994), nonlinear optics (Vladimirov et al. 2002), ferrofluids (Richter \& Barashenkov 2005) and several systems of interest in fluid mechanics, including natural doubly diffusive convection (Ghorayeb \& Mojtabi 1997; Bergeon \& Knobloch 2008), magnetoconvection (Blanchflower 1999; Dawes 2007; Lo Jacono, Bergeon \& Knobloch 2011), binary fluid convection (Kolodner 1993; Batiste \& Knobloch 2005a; Batiste et al. 2006; Mercader et al. 2011a) and plane Couette flow (Schneider, Gibson \& Burke 2010). Spatially localized oscillations known as oscillons are found in vibrating granular media (Umbanhowar, Melo \& Swinney 1996) and colloidal suspensions (Lioubashevski et al. 1999). The localized states mentioned above are typically left-right symmetric and hence do not drift. In contrast, asymmetric localized states in translation-invariant systems are expected to drift; stationary asymmetric localized states are thus generically only found in systems with spatial inhomogeneities or boundaries that trap or pin an asymmetric localized state that would otherwise be in motion. 
In the present work we use binary fluid convection to compute for the first time spatially localized states that move horizontally and study their properties, including their collisions. Binary fluid convection is well-known for supporting a large variety of different localized structures. It is therefore important to distinguish the structures we study, moving convectons, from the localized travelling waves that have been studied in this system, both experimentally and theoretically, over a number of years. Wall-attached localized travelling waves were first discovered in rectangular containers as reviewed by Kolodner, Surko \& Williams (1989) and Steinberg et al. (1989). Subsequent experiments in annular domains (Bensimon et al. 1990; Niemela, Ahlers \& Cannell 1990) revealed that localized travelling waves are an intrinsic state of the system whose existence does not depend on the presence of boundaries. Careful experiments (Kolodner 1991a) showed that such localized travelling waves in general drift, although they are very sensitive to small inhomogeneities in the experimental cell. Collisions among such drifting pulses of localized travelling waves have also been studied (Kolodner 1991b).

Localized travelling waves consist of a travelling wave state that propagates with phase speed $c_{p}$ under an envelope that is itself moving with a group speed $c_{g}$. As a result such states are quasi-periodic; their existence is related to the travelling waves created in a Hopf bifurcation from the conduction state. In contrast, the states of interest in the present work are related to steady overturning convection and hence to a steady-state bifurcation from the conduction state. The structures we study are moving steady states, and are singly periodic in time. The physics responsible for the motion is quite different, too. Moving convectons move because they are not left-right symmetric; in contrast, localized travelling wave packets move because of a non-zero group speed.

Binary fluid convection is perhaps the first fluid system where stationary spatially localized states, nowadays referred to as convectons, were observed experimentally (Kolodner 1993). The origin and properties of these states are now well established (Batiste et al. 2006; Mercader et al. 2010). In particular, it is known that when the system possesses reflection symmetry in the midplane (i.e. Boussinesq symmetry) two types of stationary convectons are present: even-parity convectons and odd-parity convectons. This observation can be used to generate moving localized states - all that is required is to break the symmetry that forces the convecton to be stationary. While this can be achieved, for example, by adding throughflow, we prefer here to focus on the so-called odd-parity convectons which are stationary because of their point symmetry, i.e. these convectons are invariant under a left-right reflection followed by a reflection in the layer midplane. Such convectons can therefore be set into motion through the simple device of breaking the midplane reflection symmetry, an effect that is easily achieved by applying different boundary conditions at the top of the layer than at the bottom. In contrast, even-parity convectons are symmetric under left-right reflection only, and hence remain stationary when the boundary conditions at the top are changed. A similar effect results from the breaking of Boussinesq symmetry, for example, by allowing for temperature-dependent diffusion coefficients or including quadratic dependence of the density on temperature.

In the system with Boussinesq symmetry odd- and even-parity convectons are organized in a so-called snakes-and-ladders structure (Burke \& Knobloch 2007) consisting of a pair of intertwined branches of localized states with opposite parity, interconnected by rung-like branches consisting of asymmetric (and hence moving) localized states. As one proceeds up this structure the localized states grow in length by nucleating new rolls on either side in such a way that the parity of the state is 
preserved. We seek here to understand how the snakes-and-ladders structure deforms when the midplane symmetry is progressively broken and the odd-parity states freed to move. We also seek to use the moving structures thereby created to study the properties of collisions between them. Since binary fluid convection is a driven dissipative system (stationary convectons are characterized by a balance between energy input from the lower boundary and energy dissipation within the convecton) we do not expect elastic collisions such as occur in completely integrable soliton-bearing systems.

In the past, simple models based on the Swift-Hohenberg equation have proved to provide reliable, albeit qualitative, predictions of the behaviour of convecton-bearing systems. In a recent paper Houghton \& Knobloch (2011) used such a model to describe the setup proposed above. Specifically, Houghton \& Knobloch (2011) showed that the loss of midplane reflection symmetry will allow odd-parity convectons to move, and described the complex sequence of transitions that must take place as the midplane symmetry is broken more and more strongly. As part of this work they also studied collisions between moving localized structures and collisions of moving structures with a stationary one. These collisions proved highly dissipative (inelastic), in contrast to collisions between solitons. In this paper, we show that the predictions of the Swift-Hohenberg model are once again remarkably accurate, despite the fact that this model has never been derived systematically as an evolution equation for any fluid system. As such our work demonstrates the utility and predictive power of this class of simplified models.

We mention that in many translation-invariant systems a left-right symmetric localized state may undergo a spontaneous symmetry-breaking instability as parameters are varied, resulting in a transition to spontaneous motion. Such bifurcations are usually referred to as parity-breaking bifurcations (Greene \& Kim 1988; Coullet, Goldstein \& Gunaratne 1989). In binary convection the termination of the branch of travelling waves on the branch of steady overturning convection provides an example of such a bifurcation involving spatially extended states. Our calculations provide another example: the bifurcation from symmetric stationary convectons to drifting asymmetric convectons. In all such bifurcations the speed of the asymmetric convecton decreases as the square root of the distance from the parity-breaking bifurcation that creates it.

All our calculations are performed for experimentally realistic parameter values and employ no-slip, no-mass flux boundary conditions at the top and bottom, and a fixed temperature boundary condition at the bottom. Cross-diffusion is included by means of the Soret effect but the Dufour effect is omitted. The low value of the (inverse) Lewis number characteristic of liquid mixtures limits the aspect ratio of the layer that is accessible to our continuation technique. Two mixtures are studied, a typical water-ethanol mixture and a typical $\mathrm{He}^{3}-\mathrm{He}^{4}$ mixture. Both systems behave in a similar way when the Boussinesq symmetry is broken although stable moving convectons are more easily found in the latter case.

This paper is organized as follows. In $\S 2$ we introduce the equations for binary fluid convection and the boundary conditions we use. Section 3 summarizes the results in the symmetric case while $\S 4$ explores the behaviour of the system as the midplane symmetry is progressively broken. Collisions between moving convectons are studied in $\S 5$ and the results compared with those from the Swift-Hohenberg model in $\S 6$. Brief conclusions follow in $\S 7$. 


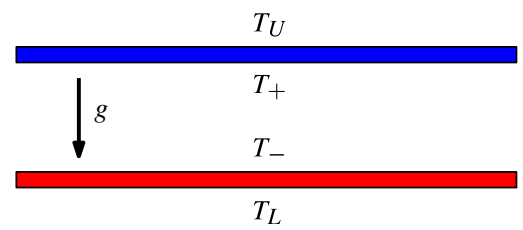

FIGURE 1. (Colour online) Sketch of the assumed geometry with partially conducting horizontal boundaries.

\section{Formulation of the problem}

Binary liquid mixtures are characterized by cross-diffusion quantified by the separation ratio $S$ that is proportional to the Soret coefficient of the mixture. When $S<0$ the heavier component (of concentration $C$ ) migrates up the temperature gradient. Thus in a layer heated from below the destabilizing temperature gradient competes with a stabilizing concentration gradient that develops in response to the heating.

\subsection{Boundary conditions}

We consider two-dimensional Boussinesq convection between boundaries responsible for the boundary conditions

$$
\begin{array}{ll}
\frac{\mathrm{d} T_{-}}{\mathrm{d} z}=-\frac{B_{-}}{d}\left(T_{L}-T_{-}\right) & \text {at } z=0, \\
\frac{\mathrm{d} T_{+}}{\mathrm{d} z}=-\frac{B_{+}}{d}\left(T_{+}-T_{U}\right) & \text { at } z=d,
\end{array}
$$

where $d$ is the layer depth, $T_{L}, T_{U}$ are the temperatures of the lower (L) and upper (U) heat baths, and $T_{-}, T_{+}$are the temperatures in the fluid next to the lower and upper boundaries (see figure 1). Here $B_{\mp}$ are the Biot numbers of the boundaries that quantify the thermal mass of each boundary relative to that of the fluid in between: conducting boundaries correspond to the limit $B \rightarrow \infty$ while an insulating boundary corresponds to $B \rightarrow 0$. Note that, by hypothesis, $T_{L}, T_{U}$ are constants independent of time, while $T_{-}, T_{+}$fluctuate in response to the motion of the fluid.

In the standard description of the Rayleigh-Bénard problem one describes the system in terms of the dimensionless temperature difference across the fluid in the conduction state, regardless of whether this state is stable or not. In this state $T^{\prime} \equiv \mathrm{d} T / \mathrm{d} z=-\Delta T^{c} / d$ everywhere, and so

$$
\Delta T^{c}=B_{-}\left(T_{L}-T_{-}^{c}\right)=B_{+}\left(T_{+}^{c}-T_{U}\right) .
$$

Here $T_{\mp}^{c}$ are the temperatures at the bottom and top of the fluid in the conduction state. Since $T_{-}^{c}-T_{+}^{c}=\Delta T^{c}$ it follows that

$$
\Delta T^{c}=\left(T_{L}-T_{U}\right) \frac{B_{+} B_{-}}{B_{+} B_{-}+B_{+}+B_{-}},
$$

and is therefore independent of the dynamics of the system, provided $T_{L}-T_{U}$ remains fixed. Moreover, in view of the equivalent relation

$$
\Delta T^{c}=\frac{B_{+} B_{-} \Delta T-d\left(B_{+} T_{-}^{\prime}+B_{-} T_{+}^{\prime}\right)}{B_{+} B_{-}+B_{+}+B_{-}},
$$


the temperature difference $\Delta T \equiv T_{-}-T_{+}$across the fluid may indeed change during the evolution of an instability but must be compensated by a corresponding variation in the temperature gradients (indicated by a prime) at the top and bottom. We propose therefore, following Clune (1993) and Prat, Mercader \& Knobloch (2000), to define a modified Rayleigh number based on the quantity $\Delta T^{c}$ (as opposed to $\Delta T$ ) as the proper bifurcation parameter for the system.

To do this we non-dimensionalize the equations in the usual way, expressing the temperature $T$ in units of $\Delta T^{c}$, distances in units of the layer depth $d$ and time in units of $d^{2} / \kappa$, where $\kappa$ is the thermal diffusivity of the fluid. We define the dimensionless control parameter by the relation

$$
R a^{\prime \prime}=\frac{\alpha g\left(T_{L}-T_{U}\right) d^{3}}{\kappa v}=R a^{\prime} \frac{T_{L}-T_{U}}{\Delta T^{c}},
$$

where $R a^{\prime}$ is defined by analogy to the usual Rayleigh number, i.e. $R a^{\prime}$ is proportional to the temperature difference across the fluid in the conduction state:

$$
R a^{\prime}=\frac{\alpha g d^{3}}{\kappa \nu} \Delta T^{c} .
$$

Note that both $R a^{\prime}$ and $R a^{\prime \prime}$ defined in this manner remain constant for fixed external conditions, in contrast to the usual Rayleigh number

$$
R a=\frac{\alpha g \Delta T d^{3}}{\kappa \nu} .
$$

In the limit $B_{ \pm} \rightarrow \infty$ the two Rayleigh numbers, $R a$ and $R a^{\prime}$, become identical. In the following we write $R=R a^{\prime}$ and use $R$ as the Rayleigh number specifying the thermal forcing of the system.

In the following we set $B_{-}=\infty$ (perfectly conducting bottom boundary) and decrease $B_{+}$from infinity to zero, thereby progressively degrading the thermal conduction properties of the upper boundary. In fact, the mathematical limit $B_{+}=0$ requires a change in the thermal forcing, from fixed temperature to fixed heat flux as described by Falsaperla \& Mulone (2010) and in greater detail by Proctor (1981). The corresponding boundary condition on the temperature fluctuation $\theta$ is $\theta_{z}=0$. In contrast, when $B_{+}=\infty$ the fluctuation vanishes on the boundary $(\theta=0)$.

\subsection{Basic equations}

In this paper we focus on two-dimensional convection. A two-dimensional formulation suffices for studies of both convectons and spatially localized waves, both of which are characteristic of binary fluid convection. Indeed, past studies have demonstrated that a two-dimensional formulation of the problem provides an excellent description of experimental observations made in relatively narrow annuli (Kolodner 1991a,b; Barten et al. 1995).

The system is described by the dimensionless equations (Batiste et al. 2001)

$$
\begin{aligned}
\boldsymbol{u}_{t}+(\boldsymbol{u} \cdot \nabla) \boldsymbol{u} & =-\nabla P+\sigma R[(1+S) \theta-S \eta] \hat{z}+\sigma \nabla^{2} \boldsymbol{u}, \\
\theta_{t}+(\boldsymbol{u} \cdot \nabla) \theta & =w+\nabla^{2} \theta, \\
\eta_{t}+(\boldsymbol{u} \cdot \nabla) \eta & =\tau \nabla^{2} \eta+\nabla^{2} \theta,
\end{aligned}
$$

together with the incompressibility condition

$$
\nabla \cdot u=0 .
$$


Here $\boldsymbol{u} \equiv(u, w)$ is the velocity field in $(x, z)$ coordinates, $P$ is the pressure, and $\theta$ denotes the departure of the temperature from its conduction profile, in units of the temperature difference $\Delta T^{c}>0$ across the layer (see (2.4)). The variable $\eta$ is defined such that its gradient represents the dimensionless convective mass flux. Thus $\eta \equiv \theta-\Sigma$, where $C=1-z+\Sigma$ is the concentration of the heavier component in units of the concentration difference that develops across the layer as a result of cross-diffusion in response to the temperature difference $\Delta T^{c}$ across it. The system is specified by four dimensionless parameters: the Rayleigh number $R$ defined in the preceding section (see (2.7)), the separation ratio $S$ that measures the concentration contribution to the buoyancy force arising from cross-diffusion, the Prandtl number $\sigma \equiv \nu / \kappa$ and the Lewis number $\tau \equiv D / \kappa$, in addition to the aspect ratio $\Gamma$ of the domain. Here $v$ is the kinematic viscosity while $D$ and $\kappa$ are, respectively, the solutal and thermal diffusivities. In writing these equations we have ignored the Dufour effect. This is appropriate for water-ethanol mixtures and for normal $\mathrm{He}^{3}-\mathrm{He}^{4}$ mixtures provided the mean temperature is sufficiently far from the $\lambda$ point of the mixture (Lee, Lucas \& Tyler 1983).

In this paper we explore the properties of the above system with no-slip impenetrable boundary conditions corresponding to

$$
u=w=\eta_{z}=0 \quad \text { on } z=0,1
$$

and the temperature boundary conditions

$$
(1-\beta) \theta_{z}+\beta \theta=0 \quad \text { on } z=1, \quad \theta=0 \quad \text { on } z=0 .
$$

In the following we refer to $\beta \equiv B_{+} /\left(1+B_{+}\right) \leqslant 1$ as the Biot number of the upper boundary. In particular $\beta=1$ when the temperature of the top boundary is fixed (perfectly conducting boundary) while $\beta=0$ corresponds to a thermally insulating boundary. Periodic boundary conditions with period $\Gamma$ are used in the horizontal. We remark that these equations remain valid in the limit $\beta \rightarrow 0$, independently of the way this limit is interpreted.

When $\beta=1$ the system (2.9)-(2.14) possesses midplane reflection symmetry. As a result stationary solutions of both even- and odd-parity are present and these satisfy $(u(x, z), w(x, z), \theta(x, z), \eta(x, z))=(-u(-x, z), w(-x, z), \theta(-x, z), \eta(-x, z))$ and $(u(x, z), w(x, z), \theta(x, z), \eta(x, z))=-(u(-x, 1-z), w(-x, 1-z), \theta(-x, 1-z), \eta(-x, 1-$ $z)$ ), respectively, relative to a suitable origin in $x$. Thus even solutions are invariant under the reflection $x \rightarrow-x$ (relative to $x=0$ ) while odd states are point-symmetric. These symmetries in turn imply that both states are stationary and hence do not drift (Batiste et al. 2006). Of course, asymmetric solutions obeying neither symmetry are also present but these are not expected to be stationary except at isolated values of the parameters of the problem.

Once $\beta \neq 1$ the midplane symmetry is lost and the only stationary solutions are those of even parity. The odd-parity states are no longer point-symmetric and hence are expected to drift, i.e. they become travelling waves.

Throughout this paper we focus on spatially localized solutions with the above properties, employing a periodic domain of aspect ratio $\Gamma=14$ as used in our earlier study of the $\beta=1$ system (Mercader et al. 2011a). This modest aspect ratio used is large enough to allow well-localized structures and permits a much more detailed study of the problem than the aspect ratio $\Gamma=60$ used by Batiste et al. (2006).

Most of the results obtained below are for water-ethanol mixtures with $S=-0.1$, $\sigma=7, \tau=0.01$. When $\beta=1$ the conduction state loses stability at a Hopf bifurcation at $R_{H} \approx 1909$. This bifurcation generates branches of travelling (TW) and standing 
waves (SW) of which the TW branch is computed by Mercader, Batiste \& Alonso (2006). We also consider the case $S=-0.5, \sigma=0.6, \tau=0.03$, appropriate for liquid $\mathrm{He}^{3}-\mathrm{He}^{4}$ mixtures (Sullivan \& Ahlers 1988; Batiste \& Knobloch 2005a,b), for which the snaking interval lies well within the region of stability of steady periodic convection and convectons are more likely to be stable, and use the resulting convectons to study convecton-convecton collisions in domains with aspect ratio $\Gamma=40$ and $\Gamma=60$.

\subsection{Numerical method}

Equations (2.9)-(2.12) are solved using a spectral code, with a Fourier expansion in the horizontal and a Chebyshev collocation method in the vertical. For the time evolution a second-order time-splitting algorithm proposed by Hugues \& Randriamampianina (1998) is used. To calculate steady solutions in an efficient manner we have adapted a pseudo-spectral first-order time-stepping formulation to carry out Newton's method (Mamum \& Tuckerman 1995), and implemented a continuation code to follow branches of stationary solutions. The travelling solutions are computed as steady solutions in a moving frame, where the speed $v$ of the wave is a nonlinear eigenvalue (Mercader et al. 2006).

Numerical continuation for $\Gamma=14, S=-0.1, \sigma=7, \tau=0.01$ typically requires 32 collocation points in the vertical direction and 650 for the Fourier pseudospectral evaluation. Direct numerical simulations of collisions in periodic domains with periods $\Gamma=40$ and $\Gamma=60$ and parameters $S=-0.5, \sigma=0.6, \tau=0.03$ require 1200 spectral points for the Fourier pseudospectral evaluation and a time step $\Delta t=10^{-4}$ in units of the vertical thermal diffusion time.

Our results are presented in the form of bifurcation diagrams showing either the dimensionless convective heat flux through the layer, $N u-1=\int_{\Gamma} w \theta \mathrm{d} x$, or the kinetic energy per unit length, $\mathscr{E}=(2 \Gamma)^{-1} \int_{\Gamma} u^{2}+w^{2} \mathrm{~d} x \mathrm{~d} z$, as functions of the Rayleigh number $R$. For moving structures the bifurcation diagrams are accompanied by plots of the drift speed $v$ as a function of $R$.

\section{The symmetric case: $\beta=1$}

In figure 2(a) we show a portion of the snakes-and-ladders structure of the pinning region in the case with midplane symmetry. One of the snaking branches corresponds to localized states with even parity, while the other shows odd-parity states. As in standard snaking the convectons grow in spatial extent as one follows either branch upward, and do so by nucleating new cells at either end. These cells are weak at the left saddle-nodes but strengthen as one follows each branch upward from the left saddle-node to the next right saddle-node. In contrast to earlier studies of this process (Batiste et al. 2006; Mercader et al. 2011a) we have also included three rung states, labelled $A_{1}, A_{2}, A_{3}$, consisting of drifting asymmetric states. Figure $2(b)$ shows one example of such a solution from each branch, corresponding to the filled circles in figure 2(a). Figure 3 shows the corresponding drift speeds. The asymmetric states also grow in length as one moves from rung to rung. Along each rung the degree of asymmetry changes continuously, from an even-parity state at one end to an odd-parity state at the other. Thus the drift speed $v$ vanishes at either end of the branch. However, in contrast to the standard snakes-and-ladders structure (Burke \& Knobloch 2007), some of the rung states terminate on the right on what would normally be the stable part of the snaking branch. This is the case for the middle rung which terminates on the even-parity states below the right saddle-node, and does 


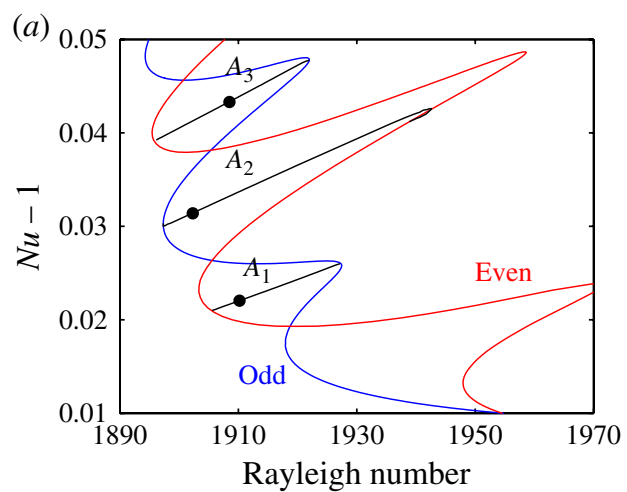

(b)

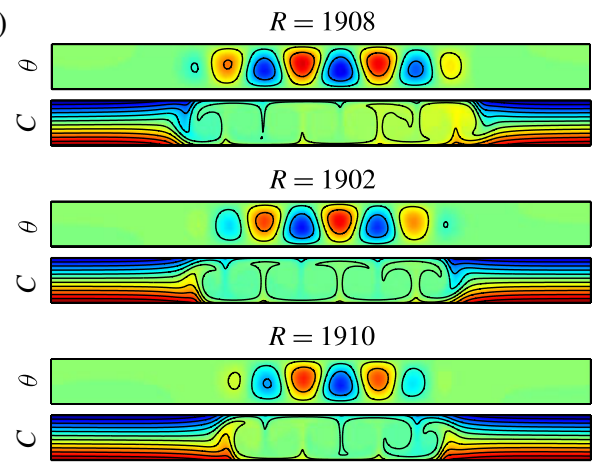

FIGURE 2. (Colour online) (a) Bifurcation diagram showing the snakes-and-ladders structure of the pinning region when $S=-0.1, \sigma=7, \tau=0.01$ and $\beta=1$. The snaking branches correspond to stationary convectons with even and odd parity, while the branches labelled $A_{1}, A_{2}$, and $A_{3}$ are rung states consisting of drifting asymmetric states. (b) Right-travelling convectons corresponding to the filled circles in the bifurcation diagram visualized in terms of contours of constant temperature fluctuation $\theta$ (upper panels) and contours of constant concentration $C$ (lower panels).

so via an additional saddle-node or fold on the branch. The net effect of this type of termination is two-fold. First, the extent of the potentially stable part of the evenparity branch is reduced, and second, the asymmetric states near the end point may acquire stability. The former effect has recently been seen in the Swift-Hohenberg equation with additional non-variational terms (Burke \& Dawes 2011). In our case we do not in fact compute the stability properties of the individual branches, and only test for stability occasionally using a time-stepping code. Figure 3 reveals one additional difference: the drift speed along the branch $A_{2}$ behaves differently from the speeds along the $A_{1}$ and $A_{3}$ branches. At the left end the branch bifurcates close to the saddle-node of the odd-parity states and, as a consequence, $v$ increases much more rapidly than the square-root behaviour along the $A_{1}$ and $A_{3}$ branches, where the corresponding bifurcation corresponds to a non-degenerate parity-breaking bifurcation. Beyond this initial phase the speed along $A_{2}$ grows linearly with Rayleigh number almost until the fold at the right, where it drops precipitously to zero. Owing to the prominent overshoot (figure 3) the drift speed along $A_{2}$ in fact changes sign just after the fold even though the solution structure does not change significantly. Evidently the nonlinear eigenvalue $v$ is exquisitely sensitive to the solution profile.

On a larger-scale (not shown) both snaking branches terminate together on a branch of periodic convection with seven pairs of cells within the periodic domain $\Gamma=14$, hereafter $P_{7}$ (Mercader et al. 2010). The termination point lies just below the fold on this branch, as in other problems of this type (Lo Jacono, Bergeon \& Knobloch 2010; Beaume, Bergeon \& Knobloch 2011). For our choice of parameters the length $\Gamma=14$ is very close to $7 \lambda_{c}$, where $\lambda_{c}$ is the critical wavelength at onset of convection. Consequently the domain chosen favours the natural wavelength of convection for the parameter values used.

It is important to observe that the snaking branches shown in figure 2(a) are in fact double (Mercader et al. 2011a). This is because there are two even-parity states, related by midplane reflection symmetry. Likewise, there are two odd-parity states, also related by midplane symmetry. The rung states have multiplicity four since each 


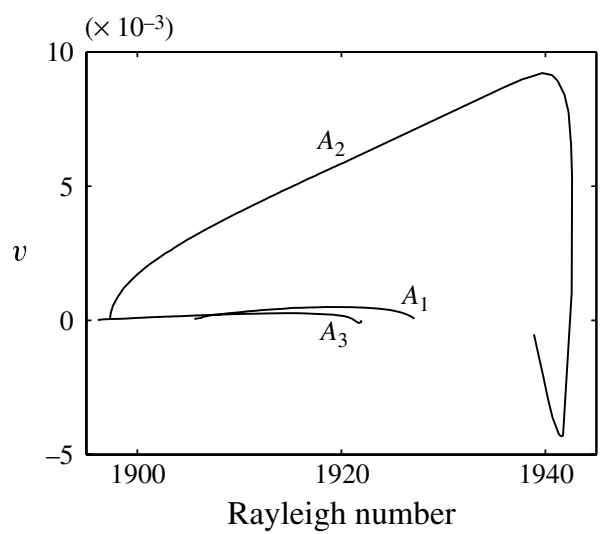

FIGURE 3. The drift speeds $v$ corresponding to the asymmetric rung states $A_{1}, A_{2}$ and $A_{3}$ shown in figure 2. States obtained by reflection in the vertical plane through the convecton centre or by reflection in the midplane drift with speed $-v$.

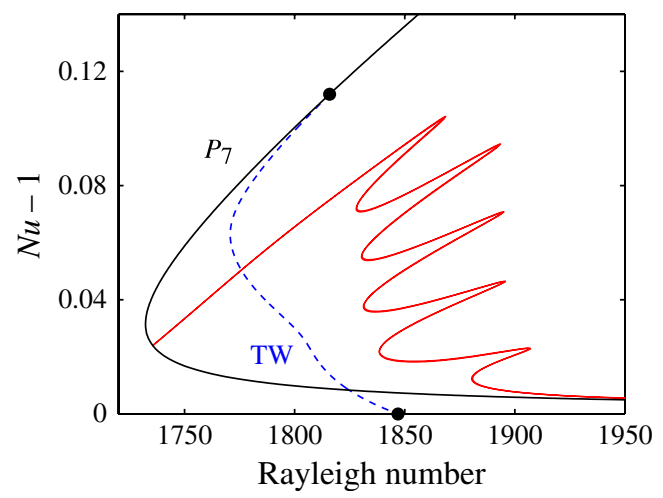

FIGURE 4. (Colour online) Bifurcation diagram for $\beta=0.95$ showing the branch of steady overturning convection with 14 rolls in the domain $\left(P_{7}\right.$, solid line), the branch of travelling waves (TW, dashed line) and a pair of snaking branches of stationary even-parity convectons.

state can be separately reflected in the midplane and left to right. Because the states are related by symmetry they are not distinguished in a bifurcation diagram such as figure 2(a). However, their multiplicity is important since it indicates that even-parity branches will split once the midplane reflection symmetry is broken, while the oddparity states will still be related by left-right reflection and so will continue to lie on a single branch in the bifurcation diagram. The rung states will likewise split into a pair of branches. These theoretical predictions are confirmed by explicit calculation in the next section.

\section{The asymmetric case}

4.1. The nearly symmetric case: $\beta=0.95$

In figure 4 we show the corresponding bifurcation diagram when $\beta=0.95$. The figure is almost identical to the corresponding figure in the symmetric case $\beta=1$ and shows 

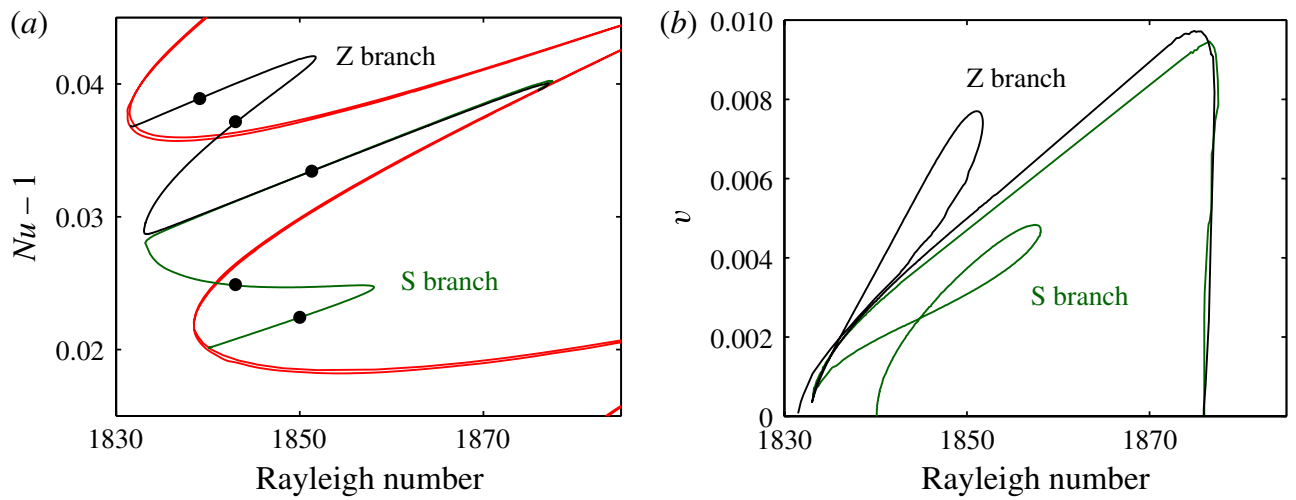

FIgURE 5. (Colour online) (a) Bifurcation diagram for $\beta=0.95$ showing a pair of branches of stationary even-parity convectons (barely distinguishable), and a pair of branches of drifting convectons labelled $\mathrm{Z}$ branch and $\mathrm{S}$ branch. (b) The drift speed along the $\mathrm{Z}$ and $\mathrm{S}$ branches. The prominent hook seen in figure 3 is now absent.

the branch of steady periodic states with 14 rolls within the domain, labelled $P_{7}$, as well as the branch of spatially periodic travelling waves, labelled TW. The TW bifurcate subcritically from the primary Hopf bifurcation (together with a branch of standing waves, not shown) and terminate in a parity-breaking bifurcation on the $P_{7}$ branch. As a result the $P_{7}$ state is unstable below this termination point but stable above it. The snaking region of even states is present in the region where the $P_{7}$ state is stable, suggesting that long, even-parity convectons will be stable. The splitting of the even-parity localized states is too small to be visible on the scale of the figure. Consequently, we show in figure $5(a)$ an enlargement of a portion of the figure corresponding to the same parameter range as figure 2(a). In addition, we have included the branches arising from the odd-parity and asymmetric states. These take two forms, hereafter referred to as $\mathrm{S}$ and $\mathrm{Z}$ branches (Houghton \& Knobloch 2011). Both form by the breakup of the pitchfork bifurcations on the odd-parity states that generate the asymmetric states along the rung states. The predicted splitting of the even-parity states is visible in the figure as is the splitting of the rung, one part of which forms part of an $\mathrm{S}$ branch while the other forms part of a $\mathrm{Z}$ branch. A careful examination shows that the $\mathrm{Z}$ branch connects the two even-parity branches produced by the splitting of the even-parity states while the $S$ branch connects an even-parity branch to itself. The stability properties along these branches are expected to be inherited from those of the $\beta=1$ case. Thus the drifting states along the middle portion of the $\mathrm{Z}$ branch are expected to be potentially stable (and likewise the solutions on the portion of the branch near the right termination point), while none of the states along the $\mathrm{S}$ branch are expected to be stable, except for those very near the upper end of the $\mathrm{S}$ branch, below the rightmost fold on the branch.

In figure $5(b)$ we show the corresponding speeds $v$. The speed vanishes near either end of the $\mathrm{S}$ and $\mathrm{Z}$ branches; it is also small in the location corresponding to the breakup of the pitchfork on the branch of odd-parity states, where it may in fact pass through zero. Along the $\mathrm{Z}$ branch the drift speed is maximum near the top right fold and again near the bottom right fold just prior to termination where it drops precipitously to zero. The situation is repeated along the $\mathrm{S}$ branch, with maximum drift speed near the top and bottom right folds. Overall, the broken midplane reflection 


\begin{tabular}{lcrcc} 
Form & \multicolumn{1}{c}{$R_{s}$} & \multicolumn{1}{c}{$v_{s}$} & $R_{l}$ & $v_{l}$ \\
$\mathrm{~S}$ & 1953.2 & $-0.63 \times 10^{-2}$ & 2135.6 & $1.26 \times 10^{-2}$ \\
$\mathrm{~S}$ & 1924.6 & $0.51 \times 10^{-2}$ & 1892.2 & $0.75 \times 10^{-2}$ \\
$\mathrm{Z}$ & 1953.2 & $0.56 \times 10^{-2}$ & 1890.6 & $0.81 \times 10^{-2}$ \\
$\mathrm{~S}$ & 1859.2 & $0.48 \times 10^{-2}$ & 1874.5 & $0.92 \times 10^{-2}$ \\
$\mathrm{Z}$ & 1857.5 & $0.77 \times 10^{-2}$ & 1875.3 & $0.97 \times 10^{-2}$ \\
$\mathrm{~S}$ & 1851.0 & $0.58 \times 10^{-2}$ & 1870.3 & $1.40 \times 10^{-2}$ \\
$\mathrm{Z}$ & 1850.7 & $1.05 \times 10^{-2}$ & 1869.9 & $1.43 \times 10^{-2}$ \\
$\mathrm{~S}$ & 1848.7 & $0.71 \times 10^{-2}$ & 1864.3 & $2.02 \times 10^{-2}$ \\
$\mathrm{Z}$ & 1845.9 & $1.19 \times 10^{-2}$ & 1863.9 & $2.04 \times 10^{-2}$ \\
$\mathrm{Z}$ & 1846.4 & $-0.66 \times 10^{-2}$ & 1855.2 & $0.48 \times 10^{-2}$
\end{tabular}

TABLE 1. Rayleigh numbers $R$ and drift velocity $v$ at the two velocity maxima along each $\mathrm{S}$ and $\mathrm{Z}$ branch when $\beta=0.95$, proceeding up the snakes-and-ladders structure in the direction of increasing $N u$. The subscripts $s$ and $l$ indicate the location of the maxima on the short $(s)$ and long $(l)$ branch segments, respectively. The type of branch, $\mathrm{S}$ or $\mathrm{Z}$, is also specified. The fourth and fifth entries correspond to figure $5(a, b)$.

has a large effect on the Rayleigh number dependence of $v$ along the segments of the $\mathrm{S}$ and $\mathrm{Z}$ branches arising from $A_{1}$ and $A_{3}$ in figure 2 but not along the segment arising from $A_{2}$ (figure 3). We emphasize that for the present parameter values the speed of the convectons generally increases as one progresses up the snaking structure, indicating that longer convectons typically travel faster than small convectons (see table 1). We note, however, that since we have kept the spatial period $\Gamma$ fixed for all our computations, longer convectons are closer to their periodically distributed neighbours, implying the existence of steeper concentration gradients between them. These gradients are in turn set up by lateral pumping as discussed already by Batiste et al. (2006). Thus the convectons do not in fact propagate through identical backgrounds and this affects their speed. Since the convecton speed $v$ is in general slow, this pumping process is essentially as described by Batiste et al. (2006); however, as $v$ increases the contours in the comoving frame may open up resulting in much more efficient, non-diffusive transport through the structure (Knobloch \& Merryfield 1992).

Figure 6(a) shows the solution profiles along the $\mathrm{Z}$ branch in figure $5(a)$ at the locations indicated by solid dots while figure $6(b)$ shows the corresponding profiles along the $\mathrm{S}$ branch. Each of these solutions drifts to the right. The top panel in figure $6(a)$ reveals the asymmetry between the fore and aft rolls that is responsible for the rightward drift, and likewise for the bottom panel in figure $6(b)$. The middle panels also exhibit fore-and-aft asymmetry but this time these states drift owing to broken midplane symmetry. Since $\beta$ is close to the symmetric case $\beta=1$ this drift is slower. However, in each case the concentration contours peak at the trailing end of the convecton suggesting that the structure is being 'pushed' by the asymmetry in the concentration field. The two solutions at $R=1851$ result from a slight splitting of an asymmetric rung state. When $\beta=1$ these states are related by the Boussinesq symmetry and hence drift in the same direction. When the Boussinesq symmetry is broken the two states are no longer related by symmetry but both continue to drift to the right. However, this time the concentration gradient across either structure is very 
(a)

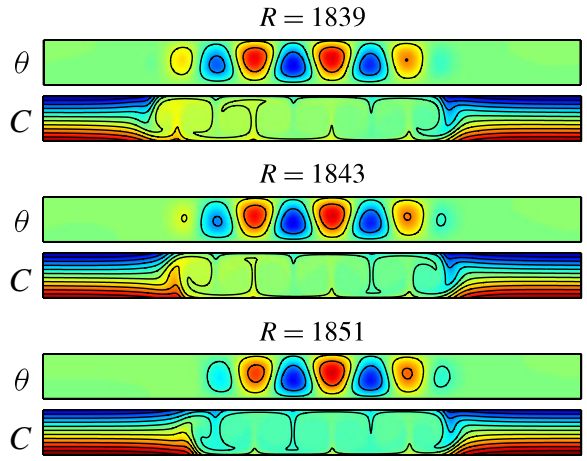

(b)

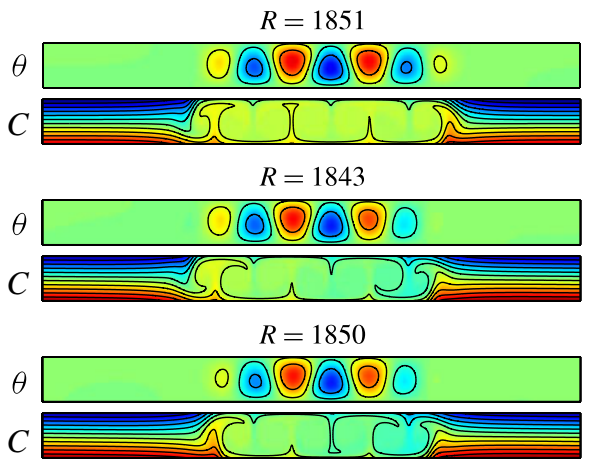

FIgURE 6. (Colour online) Contours of constant temperature fluctuation $\theta(x, z)$ and concentration $C(x, z)$ on $(a)$ the $\mathrm{Z}$ branch and $(b)$ the $\mathrm{S}$ branch at locations indicated by solid dots in figure 5(a). All solutions drift to the right and are unstable.
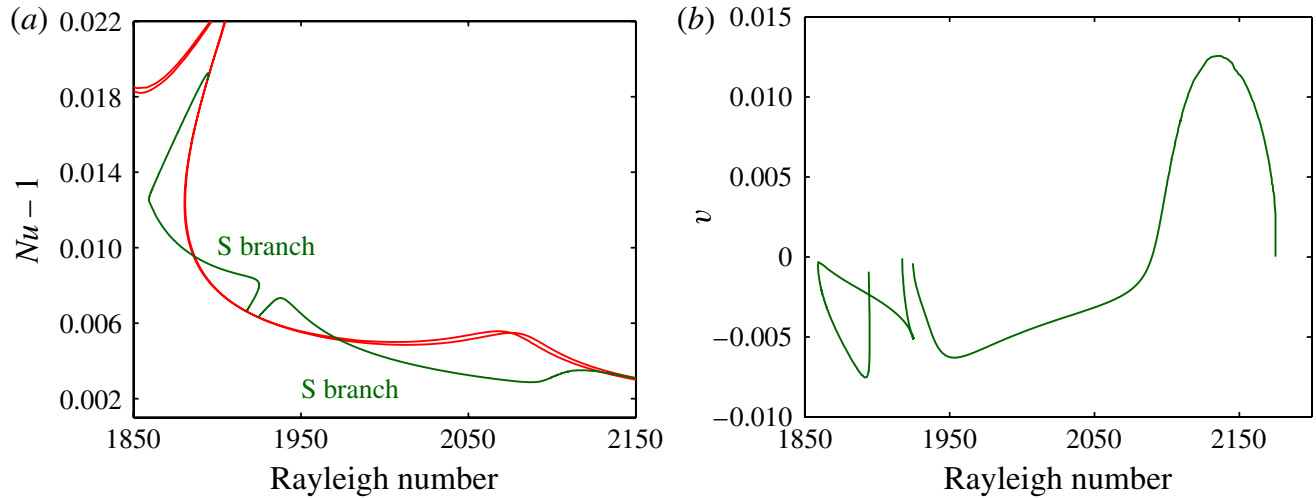

FIgURE 7. (Colour online) (a) The lower portion of the bifurcation diagram for $\beta=0.95$ showing a pair of branches of even convectons and a pair of $\mathrm{S}$ branches of drifting convectons. (b) The drift speed along the $\mathrm{S}$ branches.

weak and the leading roll rotates clockwise on the $\mathrm{Z}$ branch but counterclockwise on the $\mathrm{S}$ branch.

In figure 7 we show the corresponding behaviour along the small-amplitude part of the snaking branches. The snaking is imperfect here and the $\mathrm{S}$ and $\mathrm{Z}$ branches no longer alternate. Indeed, figure $7(a)$ reveals the presence of two consecutive $S$ branches, connecting an even-parity branch to itself. One likewise finds departures from the regular behaviour once the periodic domain is almost full, and the branches leave the snaking region (Mercader et al. 2011a).

\subsection{Intermediate values of $\beta$}

In figure $8(a)$ we show the bifurcation diagram for $\beta=0.5$. For this value of $\beta$ the splitting of the even-parity states is much larger but the $\mathrm{S}$ and $\mathrm{Z}$ branches remain much as before. Figure $8(b)$ shows the corresponding drift speeds and reveals that the maximum drift speed along the $\mathrm{Z}$ branch is now associated with the first fold on the right instead of the second fold on the right, cf. figure 5. The dominance of the first 

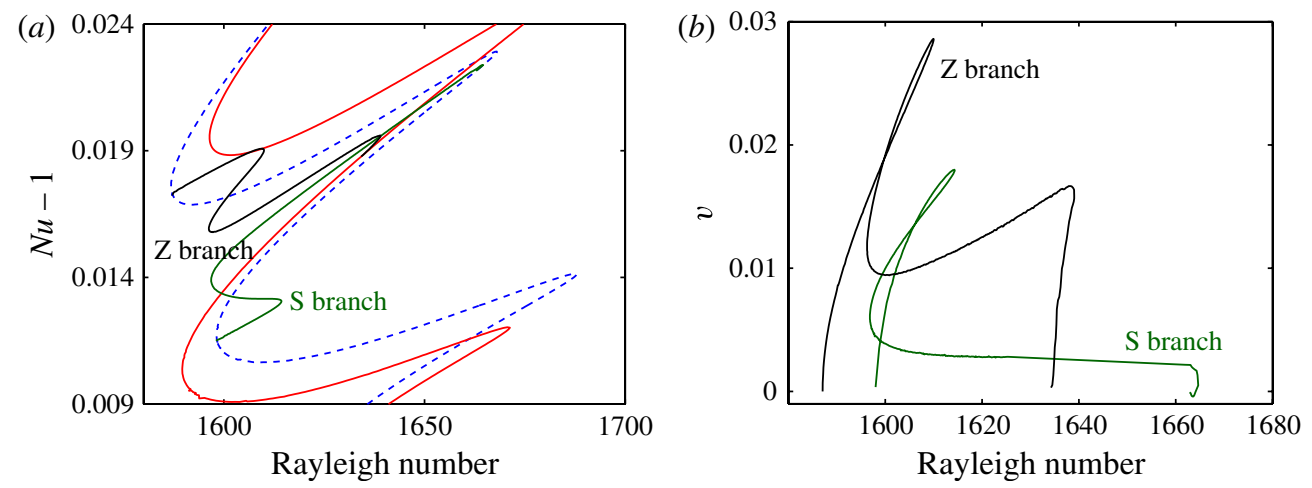

FIgURE 8. (Colour online) (a) Bifurcation diagram for $\beta=0.5$ showing a pair of branches of stationary even-parity convectons (solid and dashed lines), and a pair of branches of drifting convectons labelled $\mathrm{Z}$ branch and $\mathrm{S}$ branch. The solid (dashed) lines correspond to convectons with rising (descending) flow in the centre. (b) The drift speed along the $\mathrm{Z}$ and $\mathrm{S}$ branches.
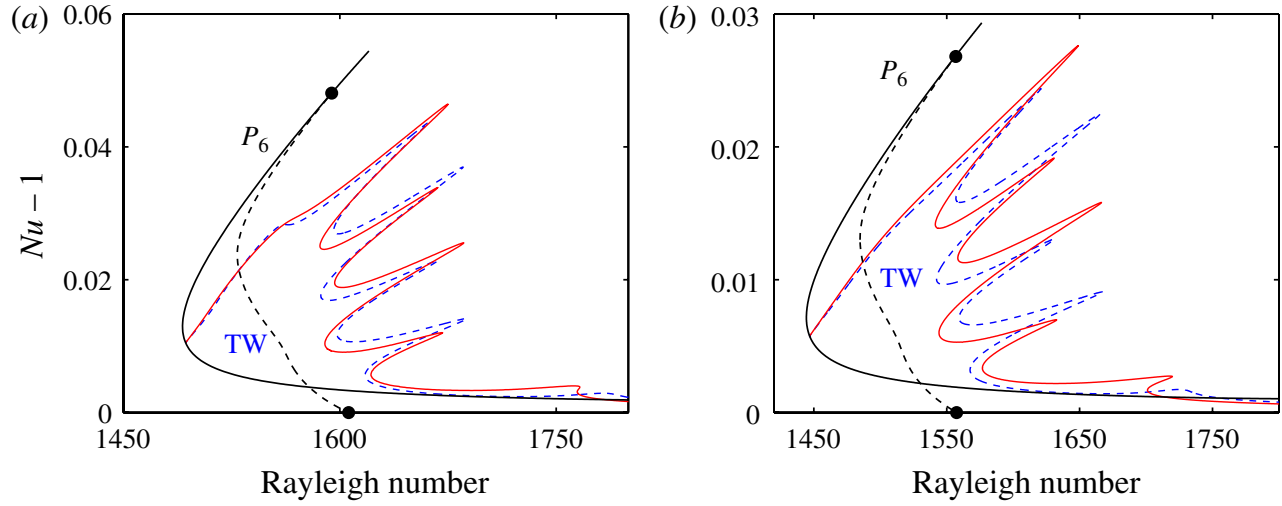

FIgURE 9. (Colour online) Bifurcation diagrams for $(a) \beta=0.5$, (b) $\beta=0.3$, showing the two branches of even-parity convectons together with the branches of periodic convection $\left(P_{6}\right)$ and travelling waves (TW). The snaking solid (dashed) lines correspond to convectons with rising (descending) flow in the centre. In both cases the $P_{6}$ branch is stable only above the filled circle.

fold develops gradually as $\beta$ decreases and is shared by both $\mathrm{Z}$ and $\mathrm{S}$ branches. At the same time the drift speed near the second fold, i.e. the point arising from the reconnection of the branches of odd and asymmetric convectons, also increases as $\beta$ decreases.

Figures $9(a)$ and $9(b)$ compare the resulting bifurcation diagrams for $\beta=0.5$ and $\beta=0.3$, respectively. The figures are quite similar although the saddle-node on the $P_{6}$ branch moves to lower Rayleigh numbers as $\beta$ decreases. At the same time the Nusselt number decreases. This is of course a consequence of the reduced conductivity of the upper boundary. This reduction manifests itself in increased wavelength of the convection - for both values of $\beta$ the convecton branches terminate on the branch $P_{6}$ with 12 rolls in the domain instead of 14 rolls. 

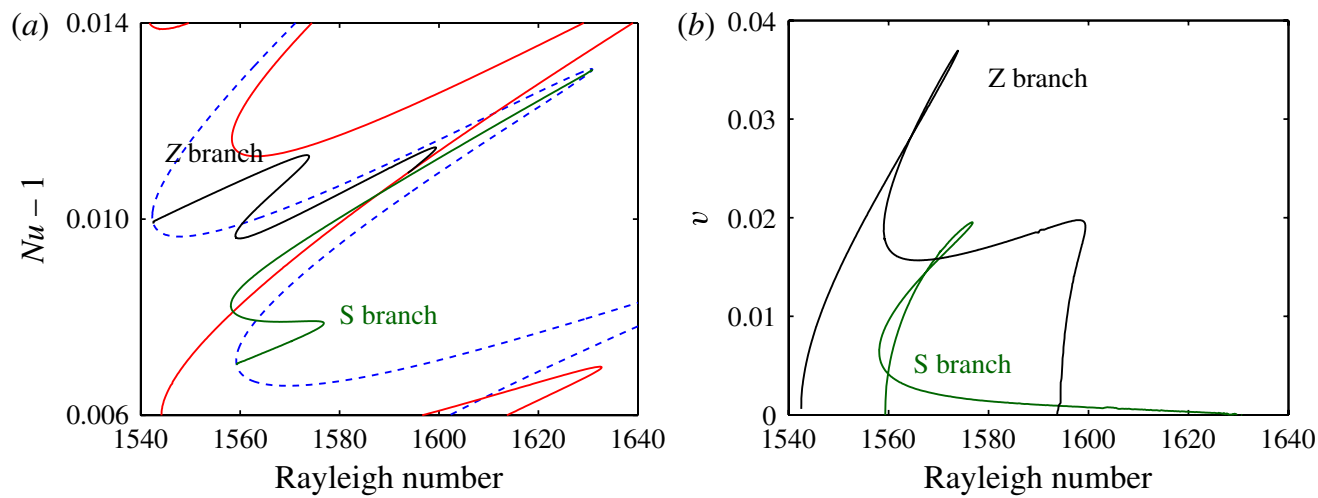

FIGURE 10. (Colour online) (a) Bifurcation diagram for $\beta=0.3$ showing a pair of branches of stationary even-parity convectons (solid and dashed lines), and a pair of branches of drifting convectons labelled $\mathrm{Z}$ branch and $\mathrm{S}$ branch. (b) The drift speed along the $\mathrm{Z}$ and $\mathrm{S}$ branches.

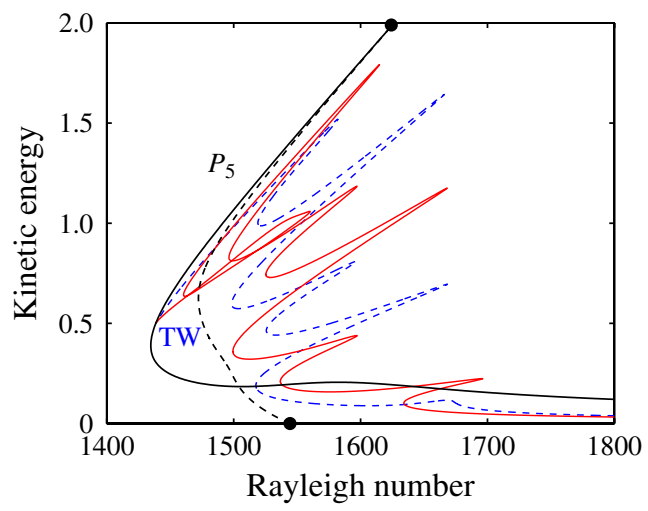

FIGURE 11. (Colour online) The bifurcation diagram for $\beta=0$, corresponding to an insulating upper boundary, showing the two snaking branches (solid and dashed lines) of even-parity convectons together with the branches of periodic convection $\left(P_{5}\right)$ and travelling waves (TW). The $P_{5}$ branch is now unstable throughout most of the pinning region.

More significantly, as $\beta$ decreases the termination of the TW branch on $P_{6}$ also moves to lower Rayleigh numbers (as does the primary Hopf bifurcation that creates it). However, the convecton branches move to lower Rayleigh numbers slightly faster, so that for $\beta=0.3$ the termination point of the TW branch now falls within the snaking region, suggesting reduced stability range for the localized states, cf. Jung $\&$ Lücke (2007). For completeness and comparison with figure $8(a, b)$, we present in figure 10 the corresponding results for the $\mathrm{S}$ and $\mathrm{Z}$ branches.

\subsection{Insulating upper boundary: $\beta=0$}

Figure 11 shows the bifurcation diagram for the case $\beta=0$. The snaking region is now almost entirely in the region of unstable $P_{5}$, the preferred number of rolls at this value of $\beta$ being 10. This is a reflection of the tendency for insulating walls to favour 

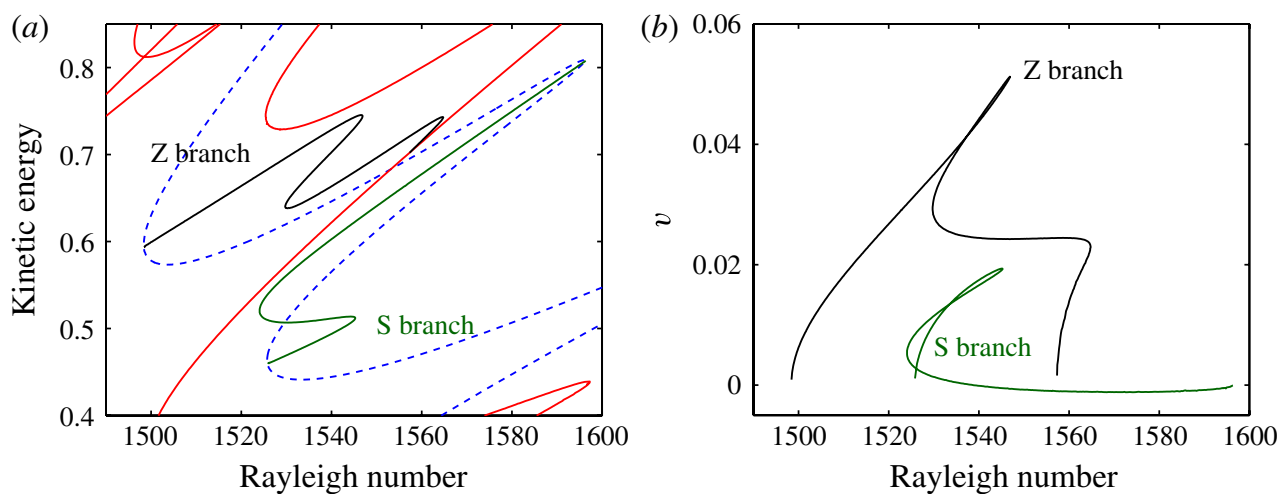

FIGURE 12. (Colour online) (a) Bifurcation diagram for $\beta=0$ showing a pair of branches of stationary even-parity convectons (solid and dashed lines), and a pair of branches of drifting convectons labelled $\mathrm{Z}$ branch and $\mathrm{S}$ branch. $(b)$ The drift speed along the $\mathrm{Z}$ and $\mathrm{S}$ branches.

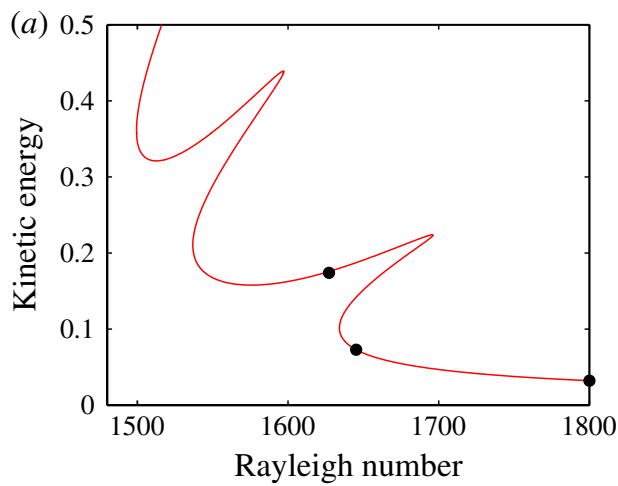

(b)

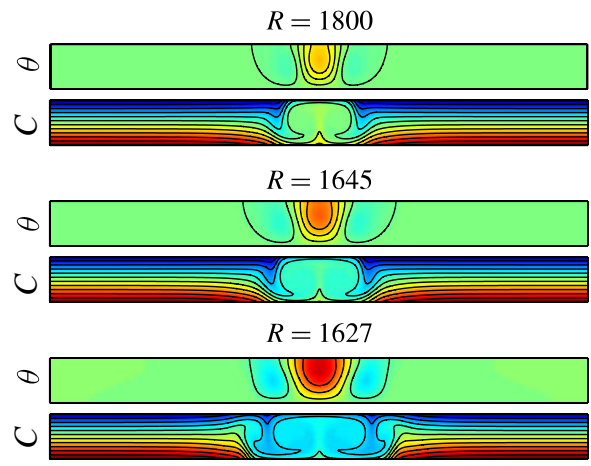

FIgURE 13. (Colour online) (a) The lower part of the bifurcation diagram for even-parity convectons when $\beta=0$. (b) The convectons corresponding to the filled circles in the bifurcation diagram visualized in terms of contours of constant temperature fluctuation $\theta$ (upper panels) and contours of constant concentration $C$ (lower panels). All three states consist of a pair of rolls with fluid rising in the centre.

larger-scale patterns (Proctor 1981; Knobloch 1989). However, even in this extreme case the $\mathrm{Z}$ and $\mathrm{S}$ branches are still present (figure 12).

It is of interest to examine the behaviour of the solution branches for this value of $\beta$ both at small amplitude (figure 13) and at large amplitude (figure 14). Figure 13(b) shows three solution profiles at increasing amplitude (decreasing Rayleigh number) showing intensification of the rising plume in the centre and the broader descending plumes on either side. In contrast, near the termination of the branch at the upper end the branch undergoes several unexpected gyrations (figure 14a). The top panel in figure $14(b)$ shows a structure that is almost periodic but as one follows the branch further the solution departs from this state and creates noticeable modulation at either end of the domain. This modulation results in a state that is dominated by three rising plumes in the centre, with a pair of weaker rising plumes on either side. Ultimately these 'defects' fill in again, and the branch terminates successfully on $P_{5}$ near its fold. 


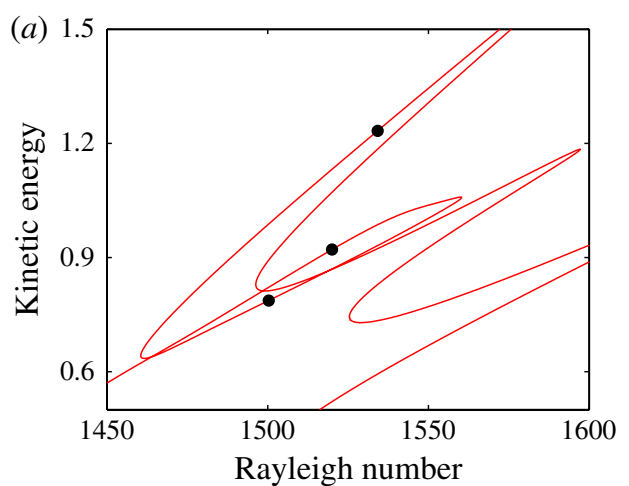

(b)

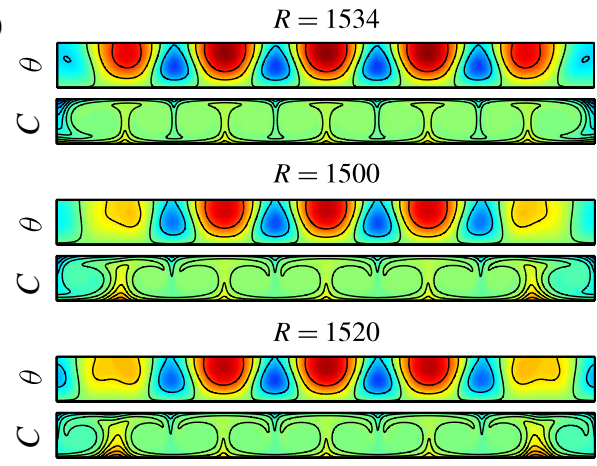

FIGURE 14. (Colour online) (a) The upper part of the bifurcation diagram for even convectons when $\beta=0$ showing an unexpected loop prior to the termination of the branch on $P_{5}$. (b) The convectons corresponding to the filled circles in the bifurcation diagram visualized in terms of contours of constant temperature fluctuation $\theta$ (upper panels) and contours of constant concentration $C$ (lower panels). The solution at $R=1520$ has adjusted the width of the descending flow near the left and right boundaries in order to terminate on the branch $P_{5}$ of periodic states (not shown).

This type of behaviour is a consequence of the finite period $\Gamma$ of the domain, and more specifically, of the incommensurability between the wavelength selected by the fronts at either end of the convectons and the prescribed period (Bergeon et al. 2008; Dawes 2009).

\section{Colliding convectons}

Now that we have been able to make convectons undergo spontaneous translation by changing the boundary condition at the top boundary we may also examine collisions between them. For this purpose we need to identify a stable drifting convecton and the work of Mercader et al. (2011b) suggests that conditions for stability with water-ethanol parameters are more easily satisfied for smaller $|S|$. However, such convectons drift slowly and we have found it advantageous to use parameters for $\mathrm{He}^{3}-\mathrm{He}^{4}$ mixtures (Sullivan \& Ahlers 1988; Batiste \& Knobloch 2005a,b) in order to obtain stable convectons that drift relatively rapidly.

Figure 15(a) shows the midplane temperature fluctuation $\theta(x, z=1 / 2)$ for a stable convecton at $R=2750$ moving to the left with speed $v=-0.016$. The panels above and below show contours of constant temperature fluctuation $\theta(x, z)$ and the associated concentration $C(x, z)$ at the initial $(t=0)$ and final $(t=100)$ times. The convecton leads with a clockwise roll, i.e. it entrains high-concentration fluid at the leading edge while entraining low-concentration fluid at the trailing edge. In this case the convecton is therefore 'pulled' by the concentration profile, i.e. the opposite of the situation shown in figure $6(a)$, middle panel, in which the fore and aft rolls also rotate in a clockwise direction but the structure drifts to the right. The solution in figure 15(a) exhibits strong concentration pumping (Batiste et al. 2006) resulting in a marked concentration gradient that extends across the convecton. However, despite its motion the temperature and concentration fields within the convecton are exactly in phase. This is in contrast to travelling waves (and localized travelling waves) in which these fields are always out of phase (Barten et al. 1995). In such states the phase difference is responsible for the motion of the wave and is in turn maintained by the motion. 
(a)
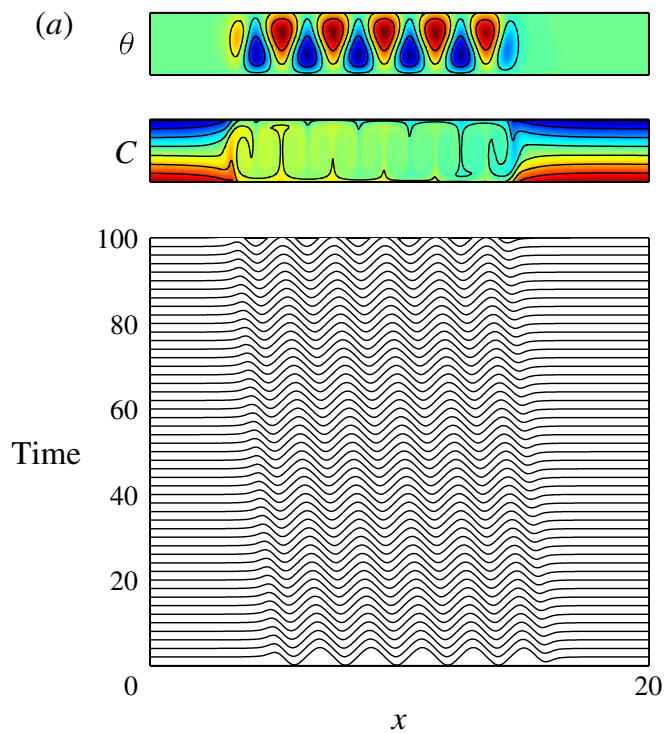

$\theta$
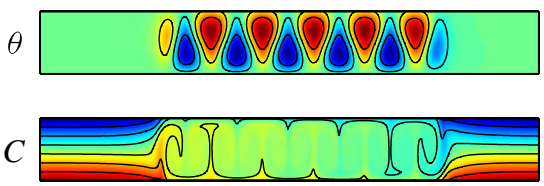

(b)
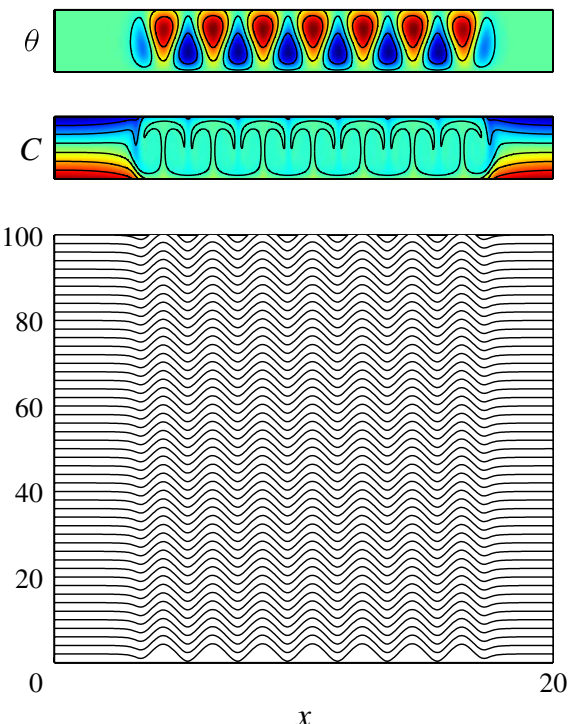

$x$
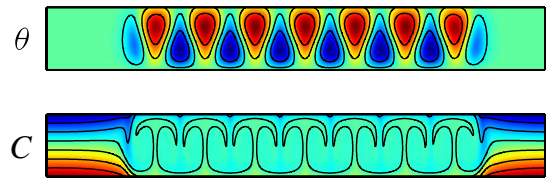

Figure 15. (Colour online) (a) A stable left-travelling convecton in a $\Gamma=20$ periodic domain at $R a=2750$ shown in the form of a space-time plot showing the midplane temperature fluctuation $\theta(x, z=1 / 2, t)$ with time increasing vertically upwards. The parameters are $S=-0.5, \sigma=0.6, \tau=0.03$ and $\beta=0.9$. (b) A coexisting stationary convecton of even parity at the same parameter values. The bottom and top panels show the profiles of the temperature fluctuation $\theta(x, z)$ and the corresponding concentration $C(x, z)$ at the initial $(t=0)$ and final $(t=100)$ times, respectively.

Figure 15(b) shows an even-parity stationary convecton for comparison. The convecton entrains cold light fluid from both sides; as a result the mean concentration within the structure is below average (figure $15 b$, lowest panel).

\subsection{Symmetric collisions}

Figure 16(a) shows a head-on collision between two identical convectons drifting in opposite directions in a periodic domain of period $2 \Gamma$ using a space-time plot. The initial condition $(t=0)$ for the left-travelling convecton is the state shown in figure $15(a)$; the right-travelling convecton at $t=0$ is obtained by left-right reflection. The collision takes place in the centre of the domain and is symmetric despite the fact that no symmetry has been imposed on the time evolution for $t>0$. When the convectons are approximately within one wavelength of one another, radiation is emitted and the convectons fall into one another. After a complex transient accompanied by additional radiation the system forms a stationary even-parity convecton with inhomogeneous wavelength distribution, followed by a relatively rapid wavelength adjustment (at $t \approx 480$ ) into the final localized state. The width of this state exceeds the combined widths of the incident convectons. The even-parity convecton that results is embedded in a background state filled with small-amplitude standing 


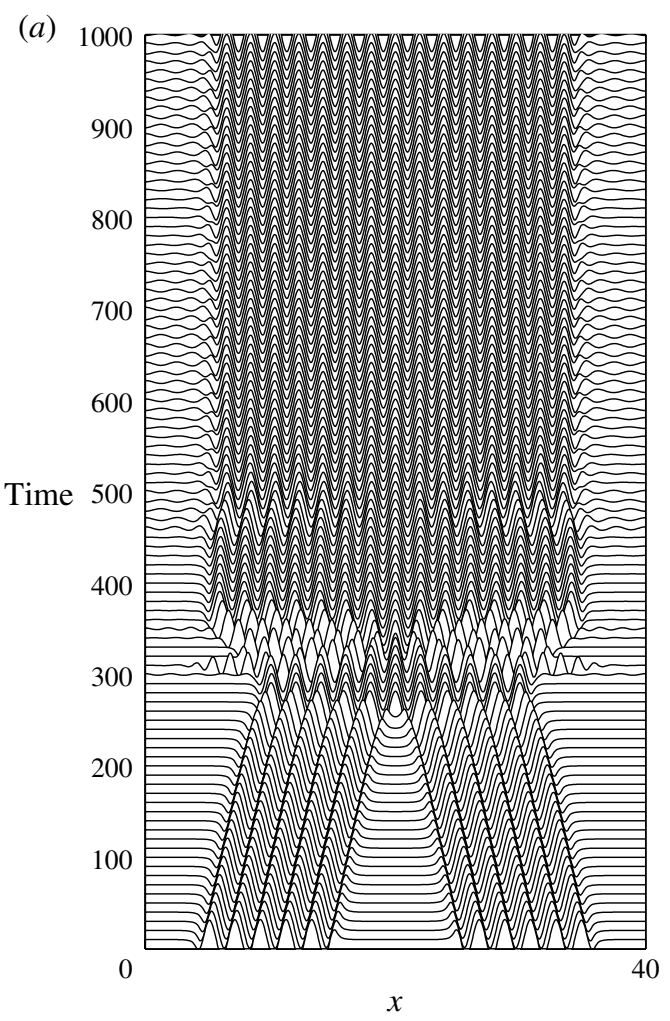

(b)
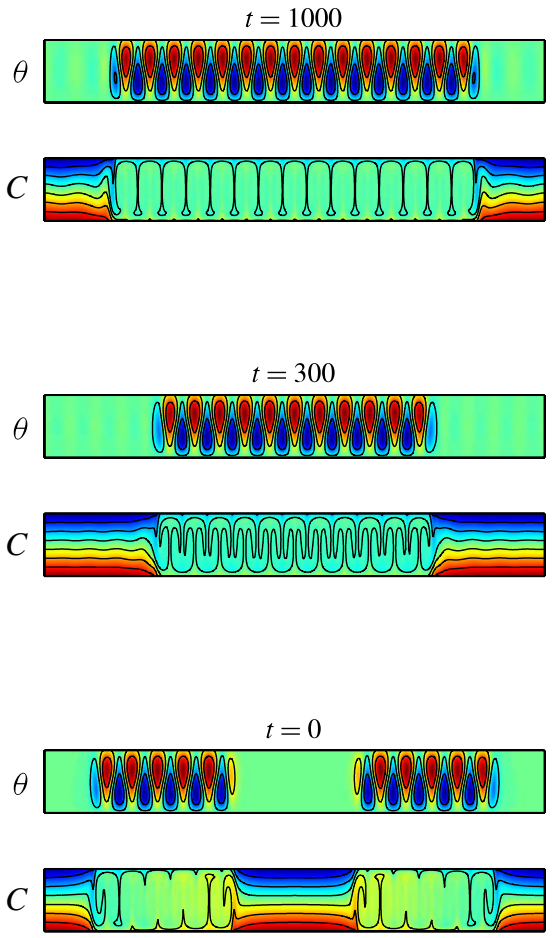

FIGURE 16. (Colour online) (a) A symmetrical collision between two identical convectons in a $\Gamma=40$ periodic domain at $R a=2750$ shown in the form of a space-time plot showing the midplane temperature fluctuation $\theta(x, z=1 / 2, t)$ with time increasing vertically upwards. The parameters are $S=-0.5, \sigma=0.6, \tau=0.03$ and $\beta=0.9$. The collision radiates waves and results in an even-parity stationary convecton whose width exceeds the combined width of the colliding convectons. (b) Snapshots of the state before $(t=0)$, during $(t=300)$ and after $(t=1000)$ the collision in terms of contours of constant temperature fluctuation $\theta$ (upper panels) and concentration $C$ (lower panels).

waves, cf. Kolodner (1993). We believe that the presence of standing waves is a consequence of the much reduced convecton-free region, a constraint that suppresses the travelling waves that are more natural in systems of this type. Indeed, the initial state at $t=0$ does not exhibit standing oscillations. Instead, small perturbations of the background evolve into travelling waves that are absorbed by the convecton as described in greater detail by Batiste et al. (2006). In this case the background state is oscillation-free despite its instability in an infinite domain.

We mention that the individual convectons used in figure 16 are stable in the half-domain $\Gamma=20$ but unstable in the larger domain $\Gamma=40$ (see $\S 5.3$ ).

\subsection{Asymmetric collisions}

Figure 17 shows the corresponding results for an asymmetrical collision between a drifting convecton and an even-parity stationary convecton. The stationary convecton entrains lower-concentration fluid at both ends, resulting in a lower than average concentration throughout the structure. This fact in turn implies that concentration is 


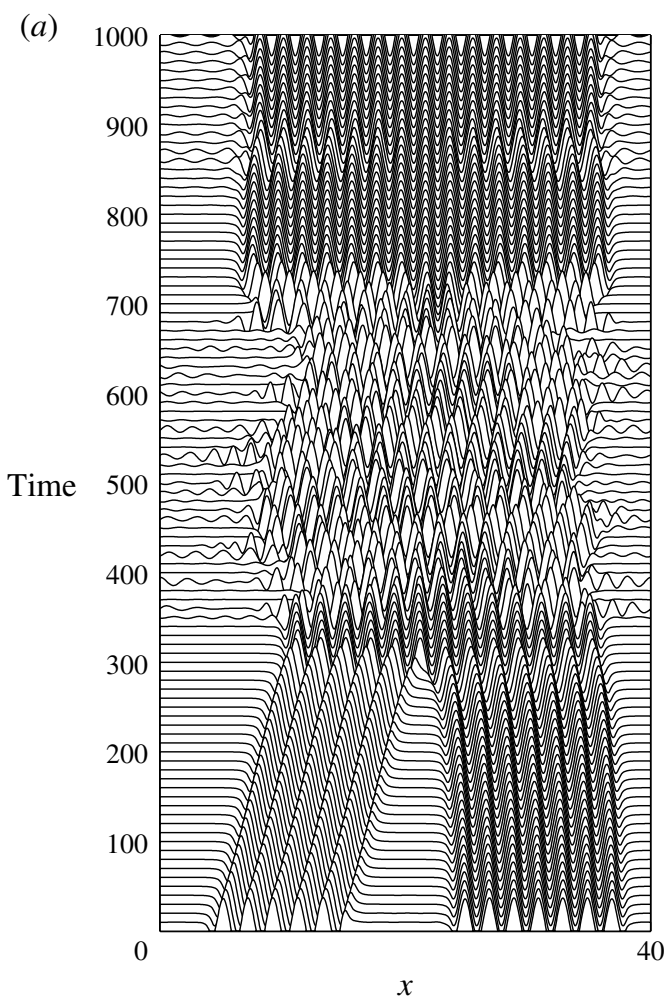

(b)
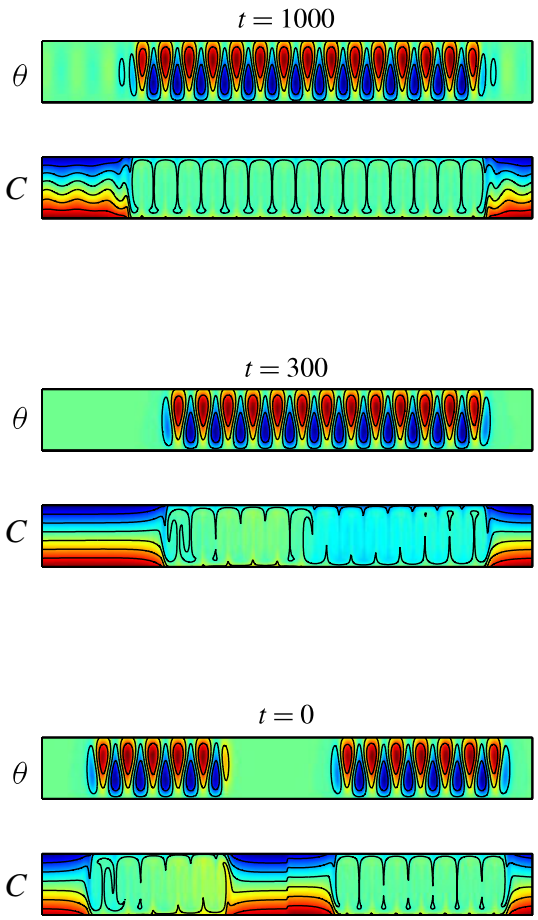

FIgURE 17. (Colour online) (a): A collision between a drifting convecton and a stationary even-parity convecton in a $\Gamma=40$ periodic domain at $R a=2750$ shown in the form of a space-time plot showing the midplane temperature fluctuation $\theta(x, z=1 / 2, t)$ with time increasing vertically upwards. The parameters are $S=-0.5, \sigma=0.6, \tau=0.03$ and $\beta=0.9$. The collision results in complex dynamics, wave radiation and ultimately in the same evenparity stationary convecton as in figure 16. (b) Snapshots of the state before $(t=0)$, during $(t=300)$ and after $(t=1000)$ the collision in terms of contours of constant temperature fluctuation $\theta$ (upper panels) and concentration $C$ (lower panels).

expelled from the convecton, setting up a step-like concentration front at $t=0$ between the incoming and target convectons. However, this concentration step dissipates rapidly, and as the incoming convecton approaches the target convecton the convectons begin to attract one another just as in the symmetric collision. This attraction is the result of a concentration gradient between the incoming convecton and the target convecton that builds up as the incoming convecton approaches the target convecton. The resulting asymmetry between the concentration on either side of the target convecton leads to attraction between the two convectons. As a result the target convecton becomes distorted and starts to drift towards the incoming convecton. This accelerates the process and the convectons fall into one another. The asymmetry of the resulting collision amplifies both the radiation that results from this process and the length of the resulting transient. However, ultimately the transient decays and the system equilibrates in the same stationary even-parity convecton as generated by the symmetric collision in figure 16. As in figure 16 this process requires the gradual elimination of the large-scale concentration inhomogeneity that is present at the moment of collision $(t=300)$. 


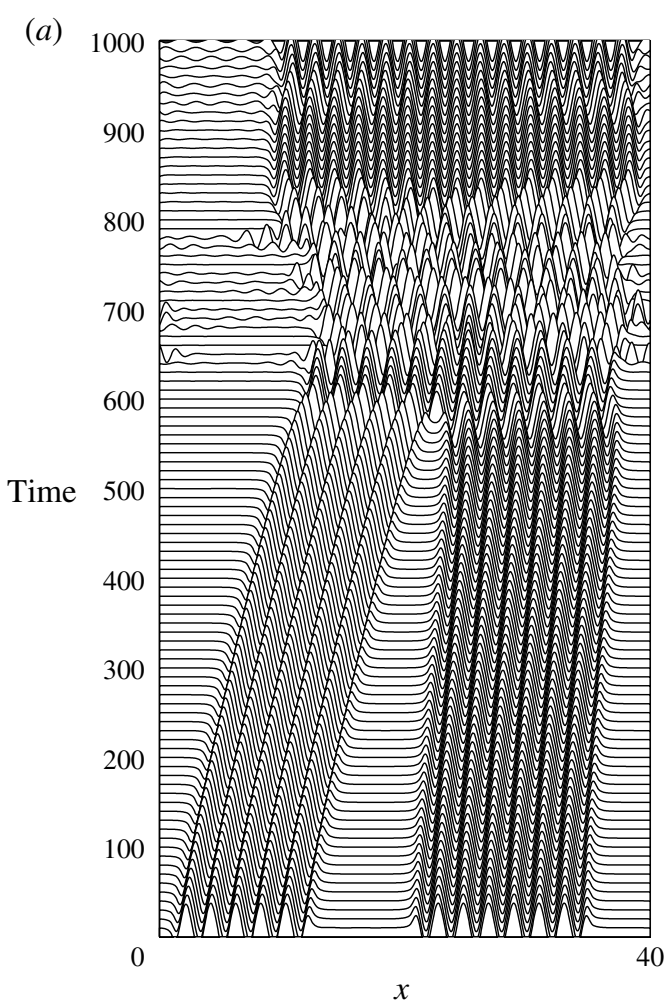

(b)
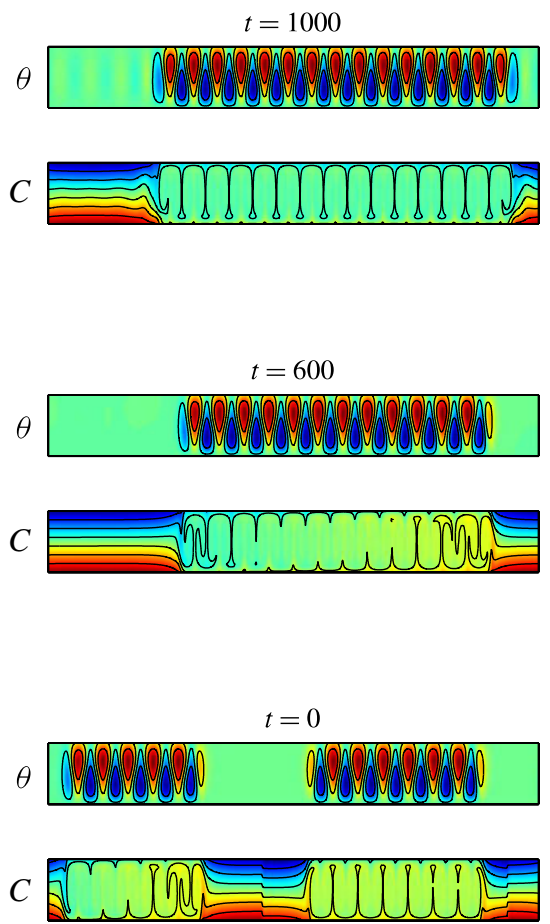

FIGURE 18. (Colour online) (a) A collision between a drifting convecton and a stationary even-parity convecton in a $\Gamma=40$ periodic domain at $R a=2750$ shown in the form of a space-time plot showing the midplane temperature fluctuation $\theta(x, z=1 / 2, t)$ with time increasing vertically upwards. The parameters are $S=-0.5, \sigma=0.6, \tau=0.03$ and $\beta=0.9$ but the stationary convecton is selected from a different segment of the branch of evenparity states than in figure 17, resulting in mutual repulsion between it and the incoming convecton. The collision results in complex dynamics, wave radiation and ultimately in the same even-parity stationary convecton as in figures 16 and 17. (b) Snapshots of the state before $(t=0)$, during $(t=600)$ and after $(t=1000)$ the collision in terms of contours of constant temperature fluctuation $\theta$ (upper panels) and concentration $C$ (lower panels).

In figure 18 we show a collision between the same drifting convecton as in figure 17 but a different even-parity stationary convecton. In this case the stationary convecton entrains higher-concentration fluid at both ends, resulting in a higher than average concentration throughout the structure. The asymmetry between the concentration to the left and right of the target convecton that builds up as the incoming convecton approaches displaces the target convecton to the right leading to a repulsion between the two convectons. This repulsion delays the collision but the eventual outcome of the collision is once again the same even-parity convecton already seen in figures 16 and 17.

\subsection{Effect of domain size}

In view of the relatively small convecton-free region that remains after the collisions shown in figures 16-18 we now examine the collision shown in figure 16 on a larger domain. We choose $\Gamma=60$ and recompute the solution starting from the same initial 
condition. Figure 19(a) shows the initial stage of the resulting evolution. We see that the instability of the background state now leads to erosion of the initial state via growing travelling waves, and its replacement by a pair of slowly drifting (almost) even convectons. These states are substantially broader and attract one another over a long time scale, resulting in a collision at $t \approx 250$ (figure 19b) at which the two 'even'-parity states coalesce into a single stationary even-parity state. This state is not stable, however, and undergoes a long chaotic transient before settling into an even broader and apparently stable even-parity state $(t=1200$, figure $19 b)$. The final state is so broad, in fact, that we may think of it as a hole in an otherwise periodic state, in other words as an anticonvecton (Mercader et al. 2011a).

The displacement of the final state relative to the original collision is most likely the result of slight asymmetry generated in the course of time integration - our integration scheme does not impose any symmetry with respect to the midpoint $\Gamma=30$. This asymmetry is greatly amplified by the rapid phase slips triggered by the continued self-compression of the structure (see the time interval $250 \lesssim t \lesssim 400$ in figure 19b), much as described by Ma, Burke \& Knobloch (2010). These set in at $t \approx 400$, creating a complex transient that allows the structure to find its preferred wavelength. These processes are not available in smaller domains, highlighting the critical role played by the aspect ratio of the domain, as remarked recently by Watanabe, Iima \& Nishiura (2012).

\section{Comparison with the Swift-Hohenberg equation}

The Swift-Hohenberg equation has proved to be a valuable and reliable model for spatially localized structures in many fluid systems (Bergeon et al. 2008; Schneider et al. 2010). In particular this equation was used to predict the snakes-and-ladders structure of the pinning region and to establish the multiplicity of stable single-pulse and multipulse localized states within this region (Burke \& Knobloch 2009; Lo Jacono et al. 2010; Beaume et al. 2011; Mercader et al. 2011a). Although the equation has some shortcomings even as a qualitative model (for example, it fails to capture the different widths of the pinning regions for odd- and even-parity localized states, or the presence of Hopf bifurcations) the equation describes faithfully the different ways in which branches of localized states terminate both in periodic domains with finite spatial period (Bergeon et al. 2008), and in closed domains with non-Neumann boundary conditions (Mercader et al. 2009, 2011a).

In a recent paper Houghton \& Knobloch (2011) used the cubic-quintic Swift-Hohenberg equation to examine the effects of breaking the symmetry $u \rightarrow-u$ of this equation. Specifically, they considered the equation

$$
u_{t}=r u-\left(1+\partial_{x}^{2}\right)^{2} u+b_{3} u^{3}-u^{5}+\epsilon u^{2}+\delta u_{x}^{2} .
$$

Here $r$ is a parameter akin to the Rayleigh number. When $\epsilon=\delta=0$ the symmetry $u \rightarrow-u$ is analogous to the midplane reflection symmetry in the present problem. The last two terms break this symmetry and thus model the effect of the Biot number measured by $\beta \neq 1$. The resulting problem is variational when $\delta=0$; otherwise it is non-variational.

Houghton \& Knobloch (2011) found that in both cases the effects of breaking the midplane reflection symmetry are similar. With increasing $\epsilon$ or $\delta$ the branch of evenparity localized states splits into two branches, one with states with maxima at $x=0$ and the other with states with minima at $x=0$. In addition the branch of odd-parity states reconnects with the branches of asymmetric localized states corresponding to 

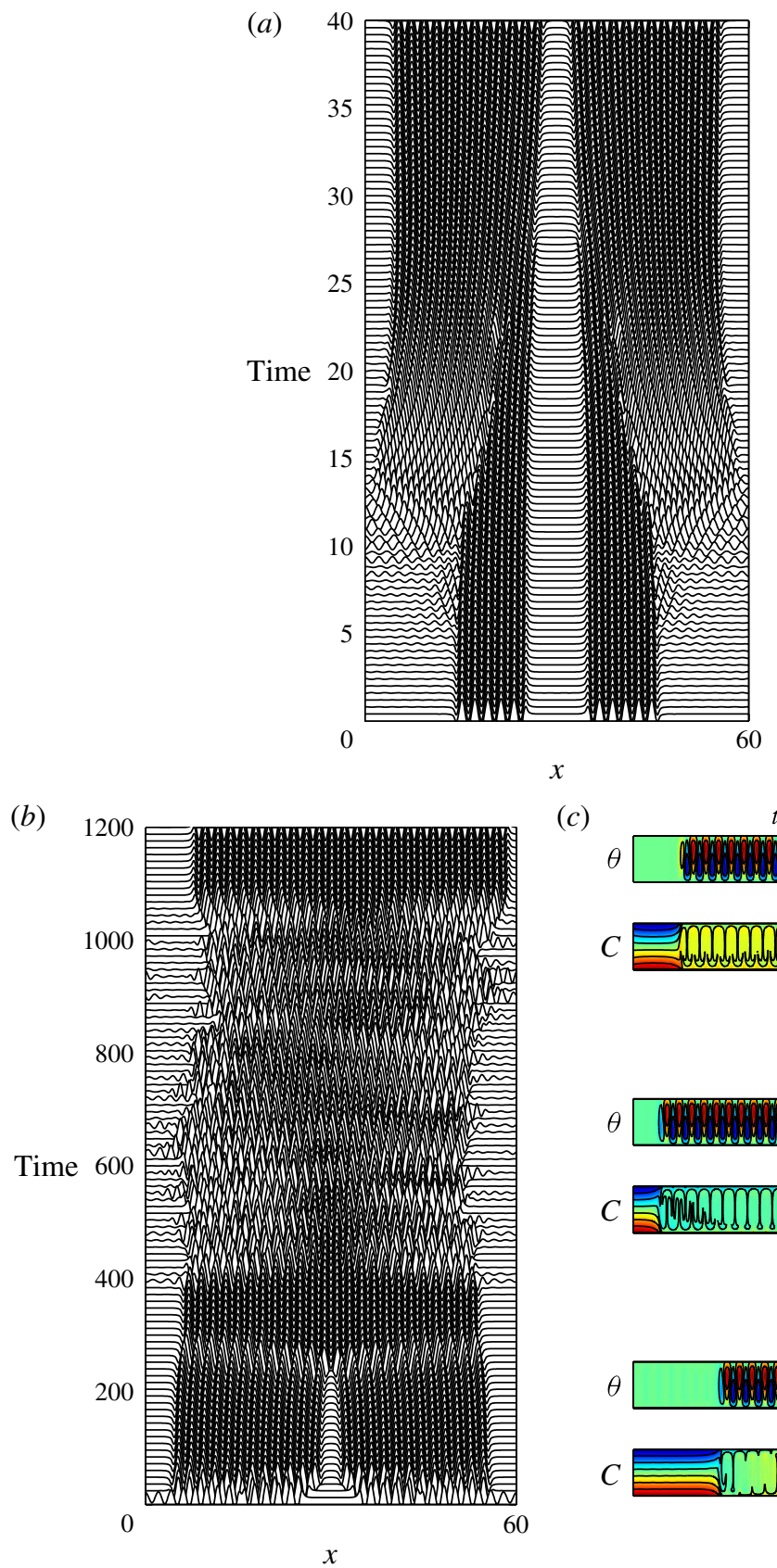

(c)

$t=1200$

$\theta$

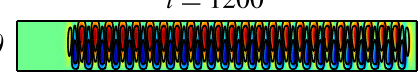

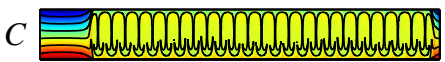
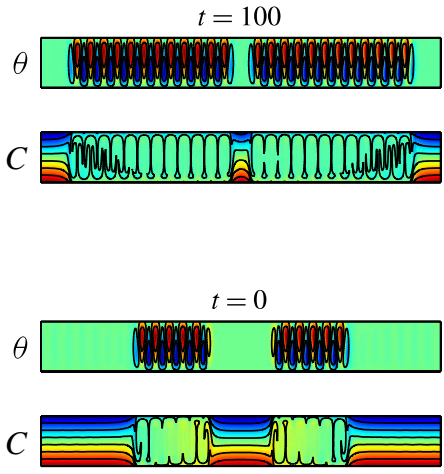

FIgURE 19. (Colour online) Same as figure 16 but for two identical convectons in a $\Gamma=60$ domain. The parameters are $R a=2750, S=-0.5, \sigma=0.6, \tau=0.03$ and $\beta=0.9$. The initial convectons are unstable to even-parity states $(a, t \approx 10)$ that attract one another, resulting in a collision $(b, t \approx 250)$. Further compression of the resulting structure creates phase slips that amplify numerical noise and generate a long chaotic transient that results in an even-parity state displaced relative to the initial collision $(b, t=1200)$. (c) Snapshots of the initial state $(t=0)$, the even states prior to collision $(t=100)$ and the final state $(t=1200)$ in terms of contours of constant temperature fluctuation $\theta$ (upper panels) and concentration $C$ (lower panels). 

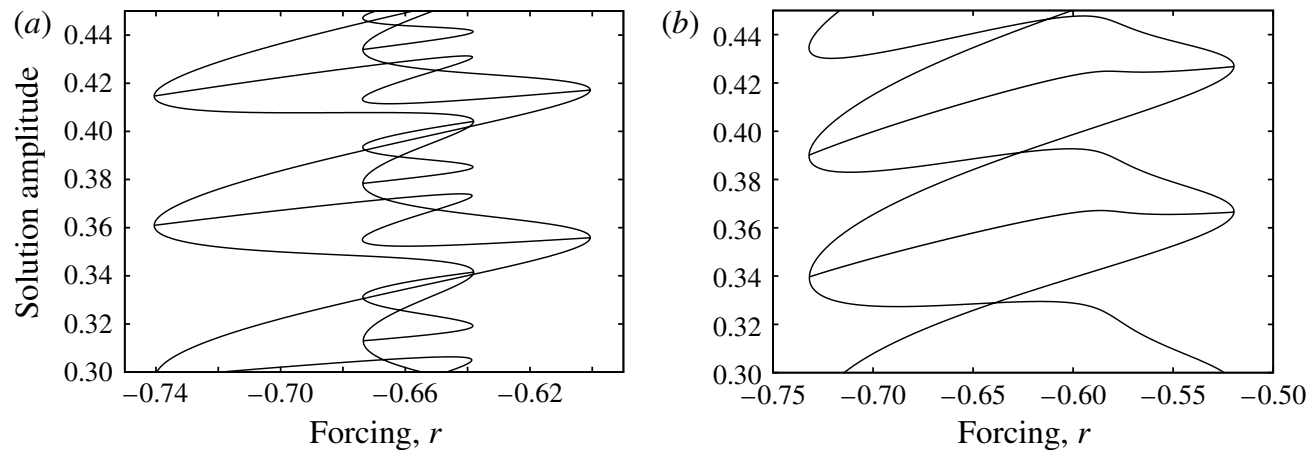

FIGURE 20. Bifurcation diagrams for the Swift-Hohenberg equation (6.1) when $b_{3}=2$, $\epsilon=0$ and $(a) \delta=0.1,(b) \delta=0.3$ for comparison with figures $8(a)$ and $10(a)$.

the rung states in the symmetric case $\epsilon=\delta=0$. These reconnection take the form of $\mathrm{Z}$ and $\mathrm{S}$ branches, and figure 20 shows two examples of the resulting bifurcation diagrams.

As already mentioned the snaking structure in the symmetric case $\epsilon=\delta=0$ differs from that identified in figure 2 in the termination of the rung states below the saddle-node on the even-parity states; this termination is preceded by a saddlenode bifurcation of the rung states. Figures $8(a)$ and $10(a)$ show that this saddlenode persists even when the midplane symmetry is substantially broken. However, aside from this effect, which may be related to the pumping effect present in the binary convection problem, the break-up of the snakes-and-ladders structure of the pinning region as $\beta$ decreases from $\beta=1$ follows precisely the scenario identified by Houghton \& Knobloch (2011) in the Swift-Hohenberg equation.

We mention one other difference between the predictions of the Swift-Hohenberg model (6.1) and the convecton problem. As shown in table 1, in the latter the maximum drift speed of broader convectons is larger than that of narrower convectons, at least for $S=-0.1, \sigma=7, \tau=0.01$ and $\beta=0.95$, in contrast to the Swift-Hohenberg model in which narrow localized structures drift faster than broad ones.

Figure 20(b) shows that with increased symmetry breaking the $\mathrm{S}$ branches eventually disappear, while the $\mathrm{Z}$ branches straighten out into rung states connecting even-parity states with maxima at $x=0$ to even-parity states with minima at $x=0$, as found in generic non-symmetric systems (Burke \& Knobloch 2006; Beck et al. 2009). In the present case the maximum symmetry breaking occurs for $\beta=0$ and as shown in figure $12(a)$ both $\mathrm{S}$ and $\mathrm{Z}$ branches are still present.

We also mention that collisions within the Swift-Hohenberg model and in binary fluid convection behave in a surprisingly similar way. In particular, when a moving convecton impacts a stationary convecton the outcome depends on whether the proximate rolls rotate in the same sense or in the opposite sense. Specifically, in the former case the structures attract while in the latter they repel. In the Swift-Hohenberg model this type of interaction is also present although it falls off exponentially the separation of the structures. In binary fluid convection the effect is much more pronounced - a consequence of a non-local interaction between the structures mediated by the concentration field associated with them. In both systems attractive interactions resulted in stationary symmetric states. In the binary mixture this 
was the case even when the structures repelled, in contrast to the Swift-Hohenberg case. Moreover, symmetric collisions in both systems were found to be attractive, possibly a result of the higher relative velocity. This observation suggests that the results reported here likely depend on the parameters employed. For example, the tendency of the structures to add rolls during coalescence could be a consequence of the Rayleigh number used, with higher Rayleigh numbers favouring roll insertion and lower Rayleigh numbers favouring roll annihilation. However, a detailed parameter study of the collision process is beyond the scope of the present paper.

\section{Conclusions}

In this paper we have used a simple and experimentally realizable recipe to generate moving convectons. The basis of our suggestion is the observation that in systems with midplane reflection symmetry odd-parity convectons will necessarily be stationary. This is a consequence of the point symmetry of these convectons, generated by a left-right reflection followed by a reflection in the layer midplane. When the latter symmetry is broken, so is the point symmetry, and the convectons generically begin to move. In contrast to odd-parity convectons even-parity states are unaffected by the loss of midplane reflection symmetry and remain stationary. The midplane symmetry is easily broken, either through the use of different boundary conditions at top and bottom or the presence of non-Boussinesq effects. We have chosen here to explore the effects of changing boundary conditions as measured by an effective Biot number $\beta$ for the upper boundary.

We have described, through the use of numerical branch following of both steady and drifting states, the transformation of the bifurcation diagram from that prevailing in the symmetric case, $\beta=1$, to its form for maximally non-symmetric boundary conditions, $\beta=0$. We have found that as $\beta$ is reduced below unity the even-parity convecton branch splits into two branches, one with upflow at the midpoint $x=0$ and the other with downflow at $x=0$. In addition, we found that the odd-parity branch reconnected with the left-right asymmetric rung states present at $\beta=1$ in two different ways, generating branches of drifting convectons we refer to as $S$ and $\mathrm{Z}$ branches according to their appearance in the bifurcation diagrams. This structure persists all the way down to $\beta=0$ and follows the behaviour identified earlier in the cubic-quintic Swift-Hohenberg equation with broken $u \rightarrow-u$ symmetry (Houghton \& Knobloch 2011).

The direction of drift cannot be readily inferred from the concentration profile across the convecton. In both cases considered, water-ethanol and $\mathrm{He}^{3}-\mathrm{He}^{4}$ mixtures, the drift direction exhibits sensitive dependence on the system parameters and more particularly on the portion of the odd-parity branch whence it came. All such drifting convectons pump concentration and we have seen that as a result convecton-convecton interactions occur via a concentration precursor. In finite domains this effect exceeds in importance the usual type of interaction between localized states which interact via exponentially decaying profiles (Burke \& Knobloch 2009) and leads to non-local interaction.

Having generated drifting convections through the simple device of changing the upper boundary condition we then performed direct numerical simulations of convecton-convecton collisions. In order to do so we chose parameter values for liquid $\mathrm{He}^{3}-\mathrm{He}^{4}$ mixtures where stable convectons are more easily obtained and used a relatively large negative separation ratio to increase their drift speed. In contrast to similar collisions performed with the Swift-Hohenberg equation (Houghton \& 
Knobloch 2011) the convecton collisions are accompanied by radiation of energy. We found that like convectons are strongly attractive when close to one another, but a drifting convecton can either attract or repel a stationary convecton depending on whether the facing roll rotates in the same direction as the incoming roll or in the opposite direction. These forces are the result of the concentration profile in the nominally convecton-free region between the two convectons. In the cases computed in detail we have found that both symmetric and asymmetric collisions were highly inelastic and yielded the same final state, a broad stationary even-parity convecton. In all cases the width of this state exceeded the sum of the widths of the incident convectons as the collision excited the nucleation of additional rolls in between. Broadly speaking these results conform to similar computations for the Swift-Hohenberg equation (Houghton \& Knobloch 2011). However, the present problem is non-variational and we have also seen that on larger domains waves can amplify if the background state is unstable and that these growing waves can substantially modify the convectons prior to their interaction.

The convecton collisions studied here differ fundamentally from collisions between localized travelling waves that are present near the primary Hopf bifurcation. These localized states consist of travelling waves under a stationary or slowly drifting envelope (Kolodner et al. 1989; Steinberg et al. 1989; Surko et al. 1991; Barten et al. 1995). States of this type can also undergo collisions (Kolodner 1991b; Iima \& Nishiura 2009; Taraut, Smorodin \& Lücke 2012; Watanabe et al. 2012) but are quite different from the drifting localized states studied here which are time-independent in the moving frame.

\section{Acknowledgements}

This work was supported in part by DGICYT under grant FIS2009-08821 and by the National Science Foundation under grant DMS-0908102.

\section{REFERENCES}

Barten, W., LÜCKe, M., Kamps, M. \& Schmitz, R. 1995 Convection in binary mixtures. II. Localized traveling waves. Phys. Rev. E 51, 5662-5680.

Batiste, O. \& KNOBLOCH, E. $2005 a$ Simulations of localized states of stationary convection in ${ }^{3} \mathrm{He}-{ }^{4} \mathrm{He}$ mixtures. Phys. Rev. Lett. 95, 244501-1-4.

Batiste, O. \& KNOBloch, E. $2005 b$ Simulations of oscillatory convection in ${ }^{3} \mathrm{He}-{ }^{4} \mathrm{He}$ mixtures in moderate aspect ratio containers. Phys. Fluids 17, 064102-1-14.

Batiste, O., Knobloch, E., Alonso, A. \& Mercader, I. 2006 Spatially localized binary-fluid convection. J. Fluid Mech. 560, 149-158.

Batiste, O., Knobloch, E., Mercader, I. \& Net, M. 2001 Simulations of oscillatory binary fluid convection in large aspect ratio containers. Phys. Rev. E 65, 016303-1-19.

Beaume, C., Bergeon, A. \& Knobloch, E. 2011 Homoclinic snaking of localized states in doubly diffusive convection. Phys. Fluids 23, 094102-1-13.

Beck, M., Knobloch, J., Lloyd, D. J. B., Sandstede, B. \& Wagenknecht, T. 2009 Snakes, ladders and isolas of localized patterns. SIAM J. Math. Anal. 41, 936-972.

Bensimon, D., Kolodner, P., Surko, C. M., Williams, H. \& Croquette, V. 1990 Competing and coexisting dynamical states of travelling-wave convection in an annulus. J. Fluid Mech. 217, 441-467.

Bergeon, A., Burke, J., Knobloch, E. \& Mercader, I. 2008 Eckhaus instability and homoclinic snaking. Phys. Rev. E 78, 046201-1-16.

Bergeon, A. \& KNObloch, E. 2008 Spatially localized states in natural doubly diffusive convection. Phys. Fluids 20, 034102-1-8. 
Blanchflower, S. 1999 Magnetohydrodynamic convectons. Phys. Lett. A 261, 74-81.

BURKe, J. \& DAwES, J. H. P. 2011 Localised states in an extended Swift-Hohenberg equation. SIAM J. Appl. Dyn. Syst. 11, 261-284.

Burke, J. \& KNOBloch, E. 2006 Localized states in the generalized Swift-Hohenberg equation. Phys. Rev. E 73, 056211-1-15.

Burke, J. \& KnObloch, E. 2007 Snakes and ladders: localized states in the Swift-Hohenberg equation. Phys. Lett. A 360, 681-688.

Burke, J. \& KNOBloch, E. 2009 Multipulse states in the Swift-Hohenberg equation. Discrete and Contin. Dyn. Syst. Suppl. 109-117.

Clune, T. L. 1993 Pattern selection in convecting systems. PhD thesis, University of California at Berkeley.

Coullet, P., Goldstein, R. E. \& Gunaratne, G. 1989 Parity-breaking transitions of modulated patterns in hydrodynamic systems. Phys. Rev. Lett. 63, 1954-1957.

DAWES, J. H. P. 2007 Localized convection cells in the presence of a vertical magnetic field. J. Fluid Mech. 570, 385-406.

Dawes, J. H. P. 2009 Modulated and localized states in a finite domain. SIAM J. Appl. Dyn. Syst. 8, 909-930.

Falsaperla, P. \& Mulone, G. 2010 Stability in the rotating Bénard problem with Newton-Robin and fixed heat flux boundary conditions. Mech. Res. Commun 37, 122-128.

Ghorayeb, K. \& Mojtabi, A. 1997 Double diffusive convection in a vertical rectangular cavity. Phys. Fluids 9, 2339-2348.

Greene, J. M. \& KIM, J.-S. 1988 The steady states of the Kuramoto-Sivashinsky equation. Physica D 33, 99-120.

Houghton, S. M. \& Knobloch, E. 2011 Swift-Hohenberg equation with broken cubic-quintic nonlinearity. Phys. Rev. E 84, 016204-1-10.

Hugues, S. \& RANDriamampianina, A. 1998 An improved projection scheme applied to pseudospectral method for the incompressible Navier-Stokes equations. Intl. J. Numer. Meth. Fluids 28, 501-521.

IIMA, M. \& NishiURA, Y. 2009 Unstable periodic solution controlling collision of localized convection cells in binary fluid mixture. Physica D 238, 449-460.

JUNG, D. \& LÜCKE, M. 2007 Bistability of moving and self-pinned fronts of supercritical localized convection structures. Europhys. Lett. 80, 14002-1-6.

KNOBloch, E. 1989 Pattern selection in binary fluid convection at positive separation ratios. Phys. Rev. A 40, 1549-1559.

KnObloch, E. \& MERRYFIELD, W. 1992 Enhancement of diffusive transport in oscillatory flows. Astrophys. J. 401, 196-205.

Kolodner, P. 1991 a Drifting pulses of traveling wave convection. Phys. Rev. Lett. 66, 1165-1168.

Kolodner, P. $1991 b$ Collisions between pulses of traveling-wave convection. Phys. Rev. A 44, 6466-6479.

Kolodner, P. 1993 Coexisting traveling waves and steady rolls in binary-fluid convection. Phys. Rev. E 48, R665-668.

Kolodner, P., Surko, C. M. \& Williams, H. 1989 Dynamics of traveling waves near onset of convection in binary fluid mixtures. Physica D 37, 319-333.

LeE, G. W., Lucas, P. \& Tyler, A. 1983 Onset of Rayleigh-Bénard convection in binary liquid mixtures of ${ }^{3} \mathrm{He}$ in ${ }^{4} \mathrm{He}$. J. Fluid Mech. 135, 235-259.

Lee, K. J., McCormick, W. D., Pearson, J. E. \& Swinney, H. L. 1994 Experimental observation of self-replicating spots in a reaction-diffusion system. Nature 369, 215-218.

Lioubashevski, O., Hamiel, Y., Agnon, A., Reches, Z. \& Fineberg, J. 1999 Oscillons and propagating solitary waves in a vertically vibrated colloidal suspension. Phys. Rev. Lett. 83, 3190-3193.

Lo Jacono, D., Bergeon, A. \& Knobloch, E. 2010 Spatially localized binary fluid convection in a porous medium. Phys. Fluids 22, 073601-1-13.

Lo Jacono, D., Bergeon, A. \& Knobloch, E. 2011 Magnetohydrodynamic convectons. J. Fluid Mech. 687, 595-605. 
Ma, Y.-P., Burke, J. \& Knobloch, E. 2010 Defect-mediated snaking: a new growth mechanism for localized structures. Physica D 239, 1867-1883.

Mamum, C. K. \& Tuckerman, L. S. 1995 Asymmetry and Hopf bifurcation in spherical Couette flow. Phys. Fluids 7, 80-91.

Mercader, I., Batiste, O. \& Alonso, A. 2006 Continuation of traveling-wave solutions of the Navier-Stokes equations. Intl J. Numer. Meth. Fluids 52, 707-721.

Mercader, I., Batiste, O., Alonso, A. \& Knobloch, E. 2009 Localized pinning states in closed containers: homoclinic snaking without bistability. Phys. Rev. E 80, 025201(R)-1-4.

Mercader, I., Batiste, O., Alonso, A. \& Knobloch, E. 2010 Convectons in periodic and bounded domains. Fluid Dyn. Res. 42, 025505-1-10.

Mercader, I., Batiste, O., Alonso, A. \& Knobloch, E. $2011 a$ Convectons, anticonvectons and multiconvectons in binary fluid convection. J. Fluid Mech. 667, 586-606.

Mercader, I., Batiste, O., Alonso, A. \& Knobloch, E. $2011 b$ Dissipative solitons in binary fluid onvection. Discrete Contin. Dyn. Syst. Suppl. S4, 1213-1225.

Niemela, J. J., Ahlers, G. \& Cannell, D. S. 1990 Localized traveling wave states in binary-fluid convection. Phys. Rev. Lett. 64, 1365-1368.

Prat, J., Mercader, I. \& Knobloch, E. 2000 Rayleigh-Bénard convection with experimental boundary conditions. In Bifurcation, Symmetry and Patterns (ed. J. Buescu, S. Castro, A. P. Dias \& I. Labouriau), Trends in Mathematics, pp. 189-195. Birkhäuser.

Proctor, M. R. E. 1981 Planform selection by finite-amplitude thermal convection between poorly conducting slabs. J. Fluid Mech. 113, 469-485.

RICHTER, R. \& BARASHENKOV, I. V. 2005 Two-dimensional solitons on the surface of magnetic fluids. Phys. Rev. Lett. 94, 184503-1-4.

SChneider, T. M., Gibson, J. F. \& BURKe, J. 2010 Snakes and ladders: localized solutions of plane Couette flow. Phys. Rev. Lett. 104, 104501-1-4.

Steinberg, V., Fineberg, J., Moses, E. \& Rehberg, I. 1989 Pattern selection and transition to turbulence in propagating waves. Physica D 37, 359-383.

Sullivan, T. S. \& Ahlers, G. 1988 Nonperiodic time dependence at the onset of convection in a binary liquid mixture. Phys. Rev. A 38, 3143-3146.

Surko, C. M., Ohlsen, D. R., Yamamoto, S. Y. \& Kolodner, P. 1991 Confined states of traveling-wave convection. Phys. Rev. A 43, 7101-7104.

Taraut, A. V., Smorodin, B. L. \& LÜCKe, M. 2012 Collisions of localized convection structures in binary fluid mixtures. New J. Phys. 14, 093055-1-23.

Umbanhowar, P. B., Melo, F. \& Swinney, H. L. 1996 Localized excitations in a vertically vibrated granular layer. Nature 382, 793-796.

Vladimirov, A. G., McSloy, J. M., Skryabin, D. V. \& Firth, W. J. 2002 Two-dimensional clusters of solitary structures in driven optical cavities. Phys. Rev. E 65, 046606-1-11.

Watanabe, T., Iima, M. \& NishiURA, Y. 2012 Spontaneous formation of traveling localized structures and their asymptotic behaviours in binary fluid convection. J. Fluid Mech. 712, 219-243. 